\title{
Subwavelength Engineering in Silicon Photonic Devices
}

\author{
Wen Zhou, Zhenzhou Cheng, Member, IEEE, Xia Chen, Member, IEEE, Ke Xu, Member, IEEE, \\ Xiankai Sun, Member, IEEE, and Hon Ki Tsang, Fellow, IEEE
}

(Invited paper)

\begin{abstract}
In the past decade there has been tremendous progress in using subwavelength-scale nanostructures with elaborately designed periodic and disordered photonic materials for applications in integrated photonics. In this paper, we review the advances in subwavelength engineering used in silicon photonic devices, with an emphasis on our own contributions on the use of subwavelength gratings and hyperuniform disordered photonic structures to attain state-of-the-art performances for near- and mid-infrared applications in fiber-chip coupling, slot waveguides for refractive-index sensing, mode conversion, wavelength filtering, integrated resonators, and ultracompact high-extinction and broadband integrated polarizers.
\end{abstract}

Index Terms-Silicon photonics, metamaterials, photonic crystals, subwavelength gratings, hyperuniform disordered photonic structures.

\section{INTRODUCTION}

$\mathrm{T}$ he reasons why silicon (Si) photonics industry has grown rapidly over the past decade are the same reasons as to why Si has been so successful in the microelectronics industry: the abundance of Si in the earth's crust, the excellent native oxide of $\mathrm{Si}$ and the cumulative investments of trillions of dollars in the microelectronics industry have produced the most advanced nanofabrication equipment for $\mathrm{Si}$ which enables low-cost highquality large Si wafers to be used for scalable high-volume manufacture of photonic integrated circuits (PICs) at high yield from Si foundries [1]. Just as the complementary metal-oxidesemiconductor (CMOS) industry was transformed

by the fabless model, the Si photonics industry is now seeing the rise of standardization in component libraries available from foundries and the availability of multi-project-wafer (MPW) services from the foundries for low-cost development of new PICs [2]. There now exist open-access Si photonic

This work was supported by Hong Kong Research Grants Council General Research Fund grant numbers 14207314 and 14205515. Wen Zhou acknowledges the support from the Postdoctoral Hub-Innovation and Technology Fund (PH-ITF). (Corresponding author: Hon Ki Tsang)

W. Zhou, X. K. Sun, and H. K. Tsang are with the Department of Electronic Engineering, The Chinese University of Hong Kong, Shatin, Hong Kong (email:wzhou@ee.cuhk.edu.hk; xksun@cuhk.edu.hk; hktsang@ cuhk.edu.hk). foundries in Europe, Asia, and North America that enable the high cost of the cleanroom fabrication equipment to be shared among different products. Thus Si photonics is becoming one of the mainstream integration platforms for optical transceivers employed in large-scale data centers [3]. With increased demand by end users, advanced modulation formats $[4,5]$ that utilize the dimensions of wavelength [6], space [7], polarization [8], and spatial modes [9] have been employed with low-cost and high-speed $\mathrm{Si}$ modulators [10]. The Si photonics platform has also attracted the development of emerging applications in integrated quantum photonics [11] for the generation and processing of entangled photons in path [12], frequency [13], time [14], and polarization [15] entangled states. Si photonics has also attracted attention for the development of integrated optical sensors for gas sensing [16] and optical frequency comb generation [17], and as a platform for optomechanics $[18,19]$.

Si photonic components are normally designed based on the formulae and software simulation tools developed for optical waveguides with only a limited choice of design parameters [20]. Because the refractive indices (RIs) are conventionally restricted to that of the available material systems. However, by leveraging on high-resolution lithography, it is possible to engineer the RI and dispersion of Si devices by employing subwavelength-scale structures [2125]. By comparing their Mie resonance wavelength $\left(\lambda_{\text {Mie }}\right)$ and Bragg resonance wavelength $\left(\lambda_{\text {Bragg }}=2 n_{\text {eff }} \Lambda\right)$, the artificial photonic structures thus created can be classified as either photonic metamaterials $\left(\lambda_{\text {Bragg }}<\lambda_{\text {Mie }}\right.$ and $\Lambda<\lambda_{\text {Mie }}$ ) or photonic bandgap (PBG) materials ( $\left.\lambda_{\text {Bragg }}>\lambda_{\text {Mie }}\right)[26,27]$, where $n_{\text {eff }}$ and $\Lambda$ are the effective RI and structural period, respectively. Photonic metamaterials are well known and have been used to engineer the effective permittivity and permeability based on the effective medium theory (EMT) in the off-resonant regime $[28,29]$ and the Mie theory with electric and magnetic dipoles [26]. PBG materials are well known for controlling the flow of light with tailored density of states [30] and special band

Z. Z. Cheng is with the Department of Chemistry, The University of Tokyo, Tokyo 113-0033, Japan (e-mail: zzcheng@chem.s.u-tokyo.ac.jp).

$\mathrm{X}$. Chen is with the Optoelectronics Research Centre, University of Southampton, Southampton SO17 1BJ, UK (e-mail: xia.chen@soton.ac.uk).

$\mathrm{K}$. Xu is with the Department of Electronic and Information Engineering, Harbin Institute of Technology (Shenzhen), Shenzhen 518055, China (e-mail: kxu@hit.edu.cn). 
structures [31], typically created by periodic photonic crystals $(\mathrm{PhC})$, but $\mathrm{PBG}$ can also be formed by certain types of disordered structures. In this paper, we review recent advances on the integrated photonic platforms which exploit the metamaterial subwavelength gratings (SWGs) [21] and hyperuniform disordered photonic structures (HUDPS) for PBG [32].

Metamaterial SWGs are periodic structures with a pitch smaller than the working wavelength and thus they can avoid Bragg diffraction effects [33]. Specifically, schematics of a SWG-patterned channel waveguide [34] are shown in Fig. 1(a). When the working wavelength $\lambda_{1}$ is larger than $\lambda_{\max }$, diffraction-free light propagation can be obtained as shown in Fig. 1(b), where $\lambda_{\max }$ is the maximum of a PBG spectral region $\left[\lambda_{\min }, \lambda_{\max }\right] . \lambda_{\text {Bragg }}$ is at the middle point of $\left[\lambda_{\min }, \lambda_{\max }\right]$. When the working wavelength $\lambda_{2}$ is within $\left[\lambda_{\min }, \lambda_{\max }\right]$, light will be Bragg reflected, and the same grating acts as a PBG material as shown in Fig. 1(c). Working in the subwavelength regime, SWG can be treated as a RI-tailorable [34], dispersiontailorable [35], anisotropic [36, 37], and birefringent metamaterial [38]. The equivalent RIs can be obtained from the $0^{\text {th }}$ - and $2^{\text {nd }}$-order EMT $[28,29]$. By adjusting the ratio of high (Si) and low index dielectric (air), the equivalent RI can be varied in a wide range. As an example, for the fundamental transverse-electric (TE) and transverse-magnetic (TM) modes, the calculated intervals of the equivalent RI are respectively $[1.1,2.6]$ and $[1.9,3.3]$ when the minimal feature size is $70 \mathrm{~nm}$ and the period is $400 \mathrm{~nm}$ in the fabricated silicon-on-insulator (SOI) SWGs. Since the first proposal of a channel waveguide patterned with a longitudinal SWG [Fig. 1(a)] by P. Cheben et $a l$. at National Research Council of Canada in 2006 [34], SWGengineered PICs and devices have attracted widespread interest and applications owing to their unique properties for use in light propagation [33], crossing [39], bending [40], coupling [41], and transformation [42]. Recently, the optical dispersion of devices were also engineered using SWG resulting in demonstrations of ultra-broadband couplers [36], polarizationindependent couplers [43], polarization beam splitters [44], sharp waveguide bends [40], and ring resonators [45]. For nearand mid-infrared (IR) applications, we developed subwavelength-engineered waveguide grating couplers (GCs) since 2009 [38, 46-51] and fully suspended waveguide platforms since 2012 [52-57], which will be reviewed in Sections II and III, respectively.

In contrast to the periodic counterpart, disordered photonic structures scatter light with speckle patterns and complex interference [58], giving rise to the Anderson localization [59], random lasing [60], and absorption enhancement [61]. Recently, photonic integrated devices patterned by the random digital-like nanostructures which were inversely designed to obtain exotic performances with compact footprints [62-66]. As a subarea of disordered photonics, HUDPS with the short-range order and statistical isotropy emerged as a new class of PBG material [32], whose concept was originally defined in the hyperuniform disordered solid (HUDS) from the perspective of point patterns' number variance by S. Torquato et al. at Princeton University in 2003 [67]. The unique properties of
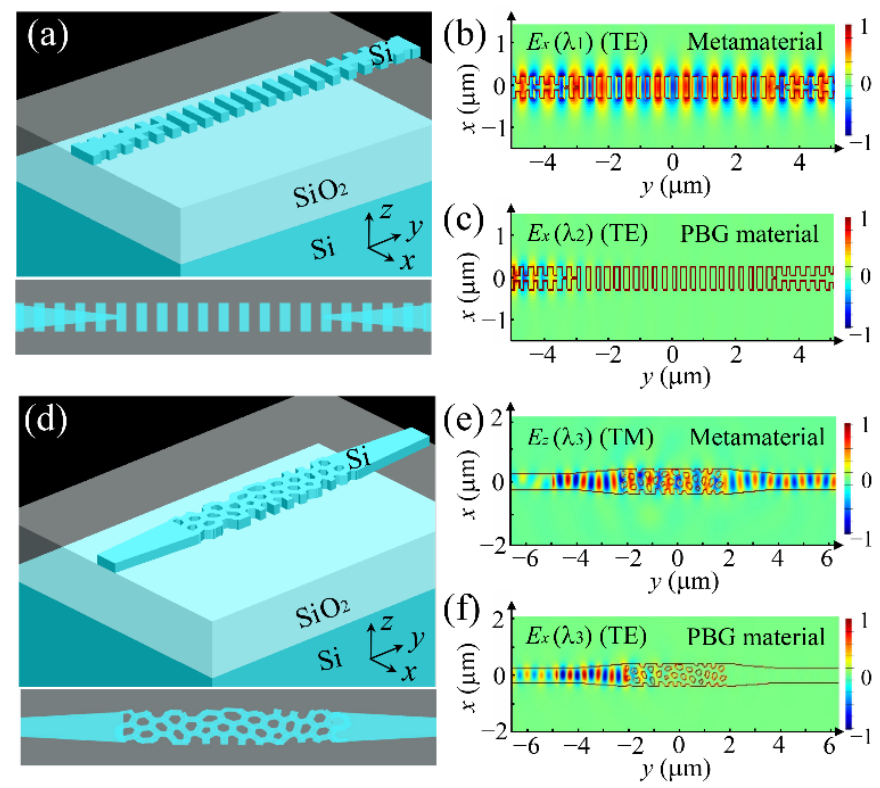

Fig. 1. Subwavelength-structured silicon photonic devices. (a) 3D schematic and top views of a channel waveguide patterned with a longitudinal SWG. (b, c) $E_{x}$ profiles of the fundamental transverse-electric (TE) mode transmitted in a SWG waveguide with $\lambda_{1}>\lambda_{\max }$ (b) and $\lambda_{2} \epsilon\left[\lambda_{\min }, \lambda_{\max }\right]$ (c). (d) $3 \mathrm{D}$ schematic and top views of a channel waveguide patterned with HUDPS. (e) $E_{z}$ profile of the fundamental transverse-magnetic (TM) mode transmitted in a HUDPS with $\lambda_{3}>\lambda_{\text {max }}^{\prime}$. (f) $E_{x}$ profile of the fundamental TE mode transmitted in the same HUDPS with $\lambda_{3} \in\left[\lambda_{\min }, \lambda_{\max }\right]$. [ $\left.\lambda_{\min }, \lambda_{\max }\right]$ and $\left[\lambda_{\text {min }}^{\prime}, \lambda_{\text {max }}^{\prime}{ }^{\prime}\right.$ are the PBG spectral regions for the TE and TM modes, respectively. Figures are reproduced from: (d-f) Ref. [69] with the permission of IEEE.

HUDPS can lead to sizeable, complete, and isotropic PBGs without the prerequisite of periodic translational order. Photon transportation in a HUDPS may exhibit scattering, diffusion, tunneling, localization, and Bragg reflection [68]. Specifically, schematics of a HUDPS waveguide [69] are shown in Fig. 1(d). Due to birefringence in a thin SOI, i. e., effective RI of the fundamental TE mode being usually larger than that of the TM mode at the working wavelength $\lambda_{3}$, a HUDPS waveguide can be designed simultaneously as a subwavelength metamaterial and a PBG material for the TM and TE modes, respectively, as shown in Figs. 1(e) and 1(f). Our group proposed HUDPS PICs as a new waveguide platform for polarization filtering, which will be reviewed in Section IV, with overall improved performances [69] compared with those integrated polarizers based on periodic PhCs [70] and SWGs [71].

\section{SubwaVelEngth-STRUCTUREd SURface GRATING COUPLERS}

Surface GCs are a fundamental waveguide device for bridging huge mode mismatch between those in a PIC and a single-mode fiber (SMF) [72]. GCs can be designed at any position of a chip and may even be erasable [73] using postfabrication trimming technology [74, 75]. With a large alignment tolerance, they provide a robust solution in optical interconnection $[76,77]$. There has been much work on GCs to produce fiber-waveguide interfaces which have broad bandwidth [47, 49], polarization insensitivity [38, 51], polarization splitting [78], perfect vertical coupling [77, 79, 80], 
wavelength demultiplexing [81-83], and space division multiplexing [84]. The basic working principle of a GC is shown in Fig. 2(a). The diffracted and incident light conserve the quasimomentum: $\beta=\mathrm{K}_{i} \cdot \sin \theta+q \cdot \mathrm{K} . \beta\left(=k_{0} n_{\mathrm{eff}}\right)$ and $\mathrm{K}_{i}(=$ $\left.k_{0} n_{\text {clad }}\right)$ are respectively the wave vectors of the diffracted light in the $\mathrm{GC}$ and incident light in the cladding, and $\mathrm{K}(=2 \pi / \Lambda)$ is the grating wave vector, where $k_{0}, n_{\text {eff, }}, n_{\text {clad, }}, \theta, q$, and $\Lambda$ are the wave vector in vacuum, effective index of the Bloch-Floquet mode propagating in a GC, RI of the cladding, off-vertical diffraction angle, diffraction order, and grating period, respectively. Thus, the phase-matching condition of a GC can be derived as [85]:

$$
k_{0} n_{\text {eff }}=k_{0} n_{\text {clad }} \sin \theta+q \cdot 2 \pi / \Lambda,
$$

Normally, $q=1$ except $q=2$ in [86] and $q=0$ in [87]. The coupling efficiency $\mathrm{CE}$ can be estimated from $(1-R) \cdot D \cdot \Gamma$, where $R, D$, and $\Gamma$ are the back-reflection loss, directionality, and overlap integral of the output diffracted field with a fiber mode, respectively [21]. The figure of merits used to evaluate the performance of a GC are usually the $\mathrm{CE}$ and 1$\mathrm{dB}$ bandwidth, but the size (chip footprint), tolerance to fabrication imperfections and requirement for additional processing steps are also important.

In 2010, an apodized one-dimensional (1D) GC demonstrated by $\mathrm{X}$. Chen et al. attained a peak coupling efficiency of $76 \%$ by utilizing the high directionality and a varying duty cycle grating for apodization of the grating strength to produce a Gaussian like diffracted mode [88]. C. Li et al. demonstrated a double-etched apodized GC with a CE of $71 \%$ [89]. In 2017, without a backside reflector, CE was pushed to a record value of $81 \%$ by using a linear apodization method [90]. CEs could reach $87 \%$ [91] and $85 \%$ [92] with a backside metal mirror to further enhance the directionality. However, the challenge is that obtaining a high $\mathrm{CE}$ relies on either small feature size (44 nm in [88], $60 \mathrm{~nm}$ in [90]) or multilayer fabrication with a relaxed feature size [89]. The 1-dB bandwidth of the conventional 1D GCs is normally $30-40 \mathrm{~nm}$ in the $1550 \mathrm{~nm}$ wavelength band [85]. 1D GCs are also polarization selective due to the birefringence of the fundamental TE and TM modes in a thin Si slab [93].

To overcome the above limitations, the grating lines in conventional 1D GCs can be replaced by periodic SWGs, which satisfy the subwavelength condition: $\Lambda<\lambda /\left(2 n_{\text {eff }}\right)$ [33]. Based on the $2^{\text {nd }}$-order EMT, equivalent RI $\left(n_{\mathrm{L}}\right)$ of a SWG can be synthesized between $n_{\text {clad }}$ and $n_{\mathrm{Si}}$. Therefore, $n_{\mathrm{L}}$ provides a new degree of freedom in the design. This concept was independently conceived by the groups at the University of Málaga and ourselves at The Chinese University of Hong Kong. In May 2009, the first design of a subwavelength grating coupler (SWGC) was proposed by R. Halir et al. with an optimized field overlap of $94 \%$ for the TM mode [94]. We had independently submitted a design for a nanohole SWGC to an MPW at the Interuniversity MicroElectronics Center (IMEC) and published the first experimental demonstration of a SWGC in September 2009 [46]. The 193nm deep-ultraviolet lithography at IMEC was sufficient to fabricate the nanohole GCs with a CE of $34 \%$ for the TE mode. Compared with the
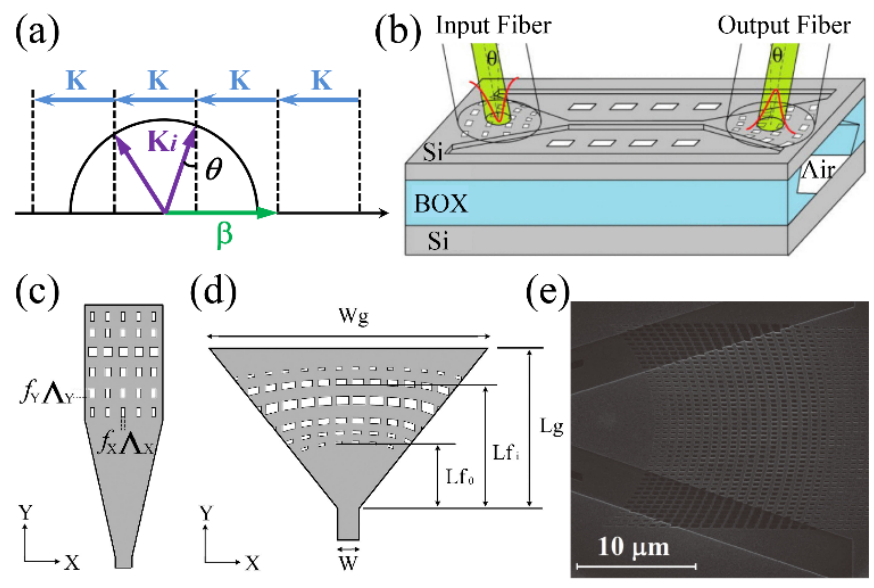

(d)

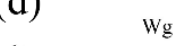

(e)

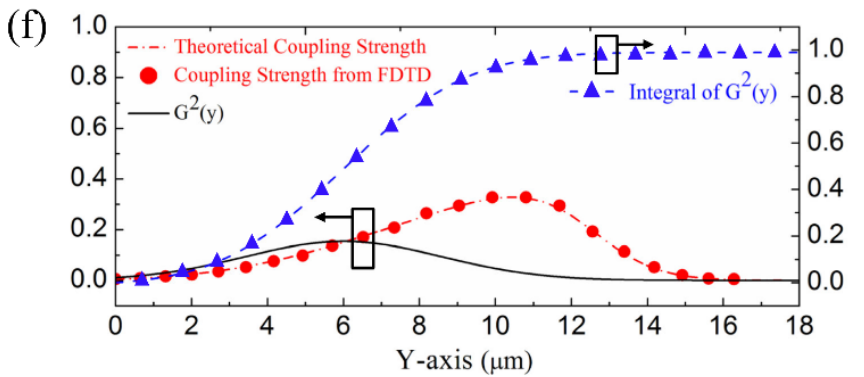

Fig. 2. Design and fabrication of the apodized focusing SWGC. (a) Working principle of a diffraction GC. (b-d) Schematics of a testing system consisting of a pair of apodized SWGCs (b), an apodized SWGC (c), and an apodized focusing SWGC (d). (e) Top-view scanning electron microscope (SEM) image of an air-cladding apodized focusing SWGC. (f) Coupling-strength engineering to achieve a Gaussian-shaped mode profile. Figures are reproduced from Ref. [48] with the permission of AIP Publishing.

conventional 1D GCs, metamaterial SWG functionalized SWGCs have the following five advantages:

1. SWG enables engineering of the RI contrast $\left(\Delta n=n_{\mathrm{Si}}-n_{\mathrm{L}}\right)$ : at the beginning of an apodized SWGC, it enables reduced back reflection [95], improved CE, and relaxed feature size $\geq 100 \mathrm{~nm}$, simultaneously [48, 94].

2. Reduced $n_{\text {eff }}$ of SWG region enables a much wider optical coupling bandwidth [47, 49].

3. Reduced dispersion in SWG broadens the 1-dB bandwidth of a SWGC [96].

4. SWG enables birefringence engineering to compensate the waveguide birefringence and enables the engineering of a polarization-independent/insensitive SWGC $[38,51]$.

5. Fully etched SWGCs also provide holes for the aqueous hydrofluoric acid (HF) to etch the buried oxide (BOX) and release the silicon device layer from substrate for mid-IR wavelength operation to avoid light absorption by the BOX [50].

\section{A. Apodized focusing SWGCs}

The empirical linear-apodization method has been employed in designing 1D GC [90] and two-dimensional (2D) SWGC [94], in which the fiber and GC diffracted mode overlap integral $\Gamma$ can be improved up to $94 \%$. We designed $\Gamma$ up to $98 \%$ by a rigorous apodization method [48]. Figures 2(b)-2(d) show schematics of a testing system consisting of a pair of apodized 
SWGCs, an apodized SWGC, and an apodized focusing SWGC with dimension labels, respectively. $\Lambda_{x}$ and $f_{x}\left(\Lambda_{y}\right.$ and $\left.f_{y}\right)$ are the period and fill factor in the $x(y)$ direction, respectively. The nondiffractive SWG grids aligned with the $x$ direction as shown in Fig. 2(c) are curved based on the phase-matching formula [97] with a focal length of $11.6 \mu \mathrm{m}$ in the $-y$ direction as shown in Fig. 2(d). Figure 2(e) shows a SEM image of an air-cladding rigorously apodized focusing SWGC for the TM-mode operation. Power attenuation in the $\mathrm{GC}$ follows $P(y)=$ $P_{0} \exp [-2 \alpha(y) \cdot y]$, where $P_{0}$ is the total power and $\alpha(y)$ is the coupling strength at the position $y$. To match a normalized Gaussian-field profile $G(y), \alpha(y)$ is given by [98]:

$$
\alpha(y)=\frac{G^{2}(y)}{2\left[1-\int_{0}^{z} G^{2}(t) d t\right]} .
$$

The red dash-dotted curve in Fig. 2(f) shows the theoretically calculated $\alpha(y)$. With the fixed $\Lambda_{x}$ of $400 \mathrm{~nm}, f_{y}$ of 0.6 , and $\theta$ of $10^{\circ}, n_{\mathrm{L}}$ was engineered to match $\alpha(y)$, whose assigned value in each diffractive unit is indicated by the red dots, while $\Lambda_{y}$ was adjusted to meet the phase-matching condition. After apodization, 2D finite-difference time-domain (FDTD) simulation predicts a $\mathrm{CE}$ of $-1.7 \mathrm{~dB}$ and a $3-\mathrm{dB}$ bandwidth of $\sim 50 \mathrm{~nm}$. The back-reflection loss is less than 14 dB. $f_{x}$ was extracted from $n_{\mathrm{L}}$ based on the $2^{\text {nd }}-$ order EMT for device fabrication. The experimentally demonstrated 3-dB bandwidth is $\sim 50 \mathrm{~nm}$ and $\mathrm{CE}$ is larger than $-3 \mathrm{~dB}$, which is improved by $\sim 1.4 \mathrm{~dB}$ compared with that of a uniform SWGC.

\section{B. Broadband SWGCS}

The dispersive diffraction angle in a $\mathrm{GC}$ working at $q=1$ and finite numerical aperture of a SMF determine the 1-dB bandwidth $\left(\Delta \lambda_{1 \mathrm{~dB}}\right)$, which is approximated by taking $d \lambda / d \theta$ from Eq. (1) [99]:

$$
\Delta \lambda_{\mathrm{ldB}}=\eta_{1 \mathrm{~dB}}\left|\frac{d \lambda}{d \theta}\right|=\eta_{\mathrm{ldB}}\left|\frac{-n_{\text {clad }} \cos \theta}{\left[n_{\text {eff }}\left(\lambda_{0}\right)-n_{\text {clad }} \sin \theta\right] / \lambda_{0}-d n_{\text {eff }}(\lambda) / d \lambda}\right|: \text {; }
$$

where $\eta_{1 \mathrm{~dB}}$ is defined by the SMF, $\lambda_{0}$ is the center wavelength. In Eq. (3), $d n_{\text {eff }}(\lambda) / d \lambda$ is normally a negative value. Therefore, $\Delta \lambda_{1 \mathrm{~dB}}$ can be enlarged by either decreasing $n_{\text {eff }}\left(\lambda_{0}\right)$ or dispersion of $-d n_{\text {eff }}(\lambda) / d \lambda$, the sum of which leads to a smaller group index $n_{g}=n_{\text {eff }}-\lambda\left(d n_{\text {eff }} / d \lambda\right)$, for the given $n_{\text {clad }}$ and $\theta$. Equation (3) can be rewritten as [100]:

$$
\Delta \lambda_{\mathrm{ldB}}=\eta_{\mathrm{ldB}}\left|\frac{-\lambda \cdot n_{\text {clad }} \cos \theta}{n_{g}-n_{\text {clad }} \sin \theta}\right| .
$$

According to Eq. (4), $\Delta \lambda_{1 \mathrm{~dB}}$ can be optimized versus $\theta$ for a given $n_{g}$. The intrinsic 1-dB bandwidth of GCs was theoretically analyzed in Ref. [99, 101, 102]. We provided the first experimental demonstration of a broadband SWGC in 2011 [47]. According to Eq. (3), reduction in $n_{\text {eff }}\left(\lambda_{0}\right)$ results in an enlarged $\Delta \lambda_{1 \mathrm{~dB}}$. Figure 3(a) shows theoretically calculated $\Delta \lambda_{\text {IdB }}$ versus $n_{\text {eff }}\left(\lambda_{0}\right)$ of SWGCs at $\lambda_{0}$ of $1.55 \mu \mathrm{m}$. This principle can be implemented by using a subwavelength pillar structure [47], such as shown in Fig. 3(b). The corresponding $n_{\text {eff }}$ is 1.7 for the fundamental TE mode, which is much smaller than that
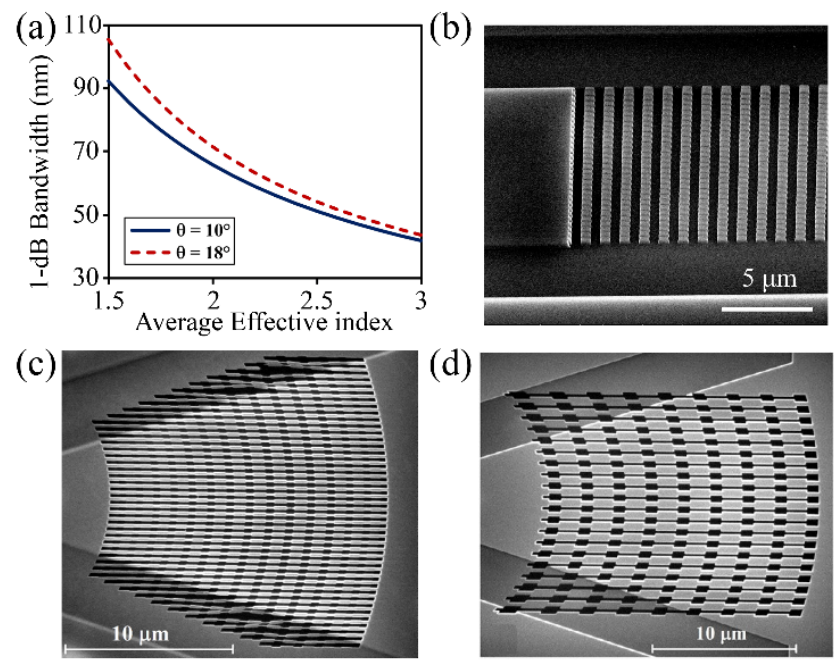

Fig. 3. Design and fabrication of broadband SWGCs. (a) Calculated 1-dB bandwidth versus $n_{\text {eff }}\left(\lambda_{0}\right)\left(\lambda_{0}=1.55 \mu \mathrm{m}\right)$. (b-d) SEM images of broadband SWGCs based on the subwavelength pillar structure (b), apodized fishbonelike structure for near-IR (c), and uniform fishbone-like structure for mid-IR (d). Figures are reproduced from: (a) Ref. [99]; (b) Ref. [47] with the permission of OSA; (c, d) Ref. [49] with the permission of OSA.

$\left(n_{\text {eff }}=3.1\right)$ in a $340-\mathrm{nm}$ Si slab. Experimentally demonstrated $\mathrm{CE}$ and 1-dB bandwidth are $-5.6 \mathrm{~dB}$ and $73 \mathrm{~nm}$, respectively. With the same principle, fishbone-like suspended focusing SWGCs were demonstrated in the near- and mid-IR regions [49], using the structures shown in Figs. 3(c) and 3(d), respectively. In each diffractive unit, there are two sets of SWGs to synthesize a low $n_{\text {eff. }} \alpha(z)$ was engineered for an apodized focusing SWGC working in near-IR. The demonstrated 3-dB bandwidth and CE are respectively $\sim 90 \mathrm{~nm}$ and $-3.5 \mathrm{~dB}$ for the TM mode at $1.534 \mu \mathrm{m}$. At the wavelength of $2.75 \mu \mathrm{m}$, a $\mathrm{CE}$ of $-5.5 \mathrm{~dB}$ was measured, and a $3-\mathrm{dB}$ bandwidth of $\sim 500 \mathrm{~nm}$ was theoretically predicted.

The contribution of $-d n_{\text {eff }}(\lambda) / d \lambda$ in increasing the $1-\mathrm{dB}$ bandwidth was experimentally investigated by wet transfer of a monolayer graphene on top of a suspended focusing SWGC [103]. According to finite-element method (FEM) calculation [104], there is $40 \%$ reduction in $-d n_{\text {eff }}(\lambda) / d \lambda$ at a Fermi level of $\sim 0.3 \mathrm{eV}$. An increase of $12 \mathrm{~nm}$ in the 1-dB bandwidth was observed after transferring graphene. The dependence of the optical bandwidth on $\eta_{\text {IdB }}$ enabled an enhanced bandwidth by simply making use of optical fibers with a larger numerical aperture [105]. In Ref. [96], $d n_{\text {eff }}(\lambda) / d \lambda$ versus the SWG period $\Lambda_{x}$ and enhancement of the bandwidth with reduced $\Lambda_{x}$ were theoretically and experimentally investigated. In Ref. [100], Eq. (4) was adopted for bandwidth estimation. $\theta$ of $25^{\circ}$ and $n_{\mathrm{g}}$ of $\sim 3.2$ ( $n_{\text {eff }} \sim 2.3$ ) were used, and the 1-dB bandwidth of $90 \mathrm{~nm}$ was achieved. Note that there is an apparent trade-off between the measured CE and $\Delta \lambda_{1 \mathrm{~dB}}$ [100]. Moreover, Q. Zhong et al. demonstrated a record 1-dB bandwidth of over $100 \mathrm{~nm}$ in a fishbone-like TE-mode SWGC with $\theta$ of $20^{\circ}$ [106].

\section{Polarization-independent/insensitive SWGCs}

2D polarization-diversity grating couplers were proposed for coupling and splitting the fundamental TE and TM modes with a low polarization-dependent loss [107, 108]. In terms of 

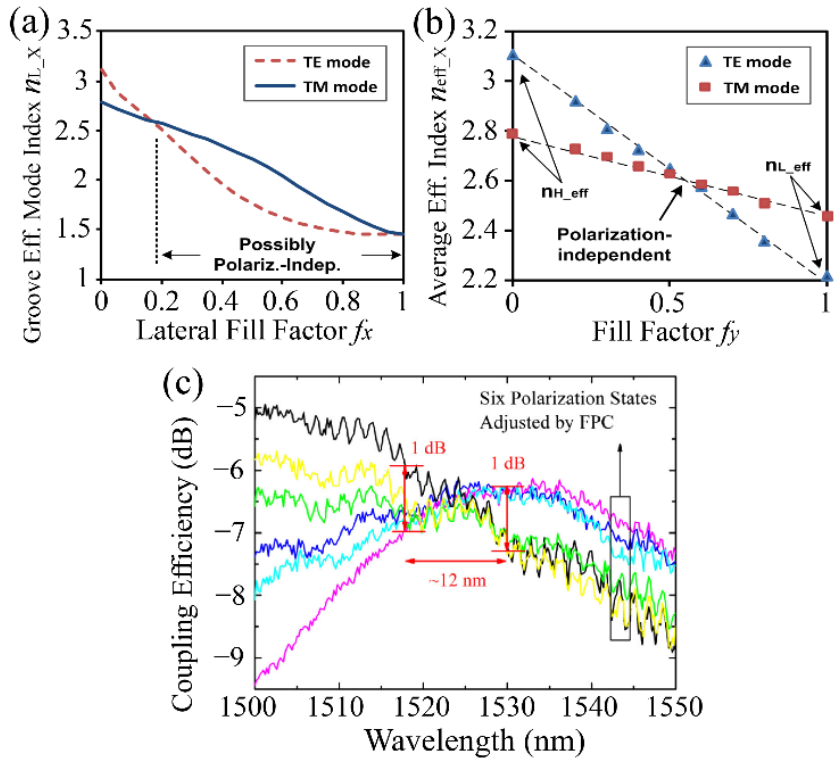

Fig. 4. Design and demonstration of polarization-independent/insensitive SWGCs. (a) Calculated effective indices of the TE and TM modes in the SWGs versus lateral fill factor $f_{x}$. (b) Calculated average effective indices in a diffractive unit versus longitudinal fill factor $f_{y}$. (c) Coupling-efficiency spectra of a polarization-insensitive focusing SWGC tested at six different polarization states. Figures are reproduced from: (a, b) Ref. [38] with the permission of OSA; (c) Ref. [51] with the permission of OSA.

the unidirectional coupling, polarization-independent GC was designed based on a micrometric-thick SOI wafer due to low birefringence [86]. However, $n_{\text {eff }}$ of the fundamental TE mode is much larger than that of the TM mode in a conventional $1 \mathrm{D}$ GC on thin SOI wafers. Metamaterial SWGs were utilized to reduce the polarization-dependence by our group in 2011 [38]. Basically, the effective RIs of the fundamental TE and TM modes in a GC should be designed with $n_{\text {eff_TE }}=n_{\text {eff_TM. }} n_{\text {eff_ } \mathrm{X}}$ $\left(\mathrm{X}=\mathrm{TE}\right.$ or TM) is calculated from $n_{\mathrm{L}_{-} \mathrm{X}} \cdot f_{y}+n_{\mathrm{H}-\mathrm{X}} \cdot\left(1-f_{y}\right)$ based on the coupled-mode theory, where $n_{\mathrm{L}_{-} \mathrm{X}}$ and $n_{\mathrm{H}_{-} \mathrm{X}}$ are the effective RIs of the fundamental TE or TM mode propagating in the SWG and Si slab waveguides, respectively [38]. In a SWG, $n_{\mathrm{L}_{-} \mathrm{TM}}>n_{\mathrm{L}_{-} \mathrm{TE}}$ can be achieved as shown in Fig. 4(a), while $n_{\mathrm{H}_{-} \mathrm{TM}}$ is smaller than $n_{\mathrm{H}_{-} \mathrm{TE}}$ in a thin Si slab. Therefore, $n_{\text {eff_TE }}=n_{\text {eff_TM }}$ can be realized by adjusting $f_{y}$ as shown in Fig. 4(b). Next, $\Lambda_{y}$ should be adjusted to achieve a diffraction angle $\theta$ according to Eq. (1). The designed polarization-independent SWGC has a predicted CE of $40 \%$. In Ref. [51], $\Delta n_{\text {eff }}=n_{\text {eff_TM }}$ - $n_{\text {eff_TE }}$ can be reduced by varying $f_{x}$ in a focusing SWGC, which were experimentally demonstrated with a minimal separation of $\sim 32 \mathrm{~nm}$ between the peak coupling wavelengths of the TE and TM modes. Figure 4(c) shows a polarizationinsensitive region with a $\mathrm{CE}$ of $-6.5 \mathrm{~dB}$ around the wavelength of $1525 \mathrm{~nm}$, and there is a $12-\mathrm{nm}$ spectral bandwidth with $<1$ $\mathrm{dB}$ polarization dependent loss.

\section{Focusing dual-wavelength-band (DWB) SWGC}

In 2018, we proposed a new type of surface GC, which couples TE-polarized light in two different wavelength bands centered at $\left(\lambda_{1}, \lambda_{2}\right)$, with a tailorable wavelength separation $\left(\Delta \lambda=\lambda_{2}-\lambda_{1}\right)$ from a SMF into a PIC with an identical $\theta[109]$.
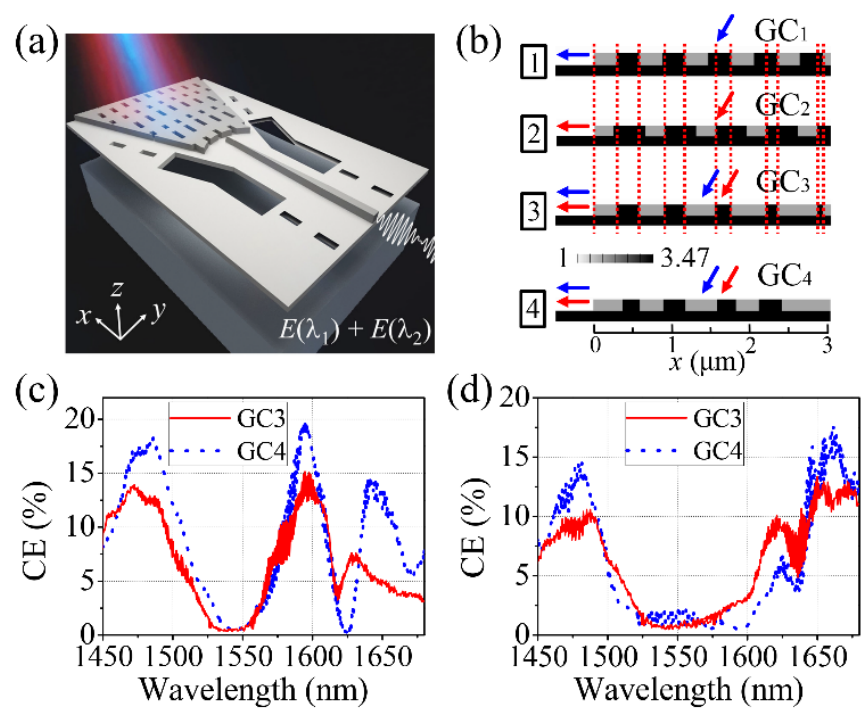

Fig. 5. Design and demonstration of focusing DWB SWGCs. (a) 3D overview of a focusing DWB SWGC based on a SOI suspended-membrane waveguide (SMW) platform. (b) Steps (1)-(4) for designing combined and optimized DWB SWGCs $\left(\mathrm{GC}_{3}\right.$ and $\left.\mathrm{GC}_{4}\right)$ from uniform single-wavelength-band $\mathrm{GC}_{1}$ and $\mathrm{GC}_{2}$. (c, d) Measured coupling-efficiency spectra of the $\mathrm{GC}_{3}$ and $\mathrm{GC}_{4}$ working at $(1486.0,1594.5) \mathrm{nm}(\mathrm{c})$ and $(1481.5,1661.5) \mathrm{nm}(\mathrm{d})$. Figures are reproduced from Ref. [109] with the permission of OSA.

The focusing DWB SWGC was designed on a SOI suspendedmembrane waveguide (SMW) platform [52], which is illustrated in Fig. 5(a). Figure 5(b) shows the methodology used to design the DWB SWGCs: SWGs in the two singlewavelength-band $\mathrm{GC}_{1}$ and $\mathrm{GC}_{2}$ are combined to form a $\mathrm{GC}_{3}$ $\left(\mathrm{SWG}_{\mathrm{GC} 1} \cup \mathrm{SWG}_{\mathrm{GC} 2}=\mathrm{SWG}_{\mathrm{GC} 3}\right)$, then the figure of merit defined by $\mathrm{CE}\left(\lambda_{1}\right) \cdot \mathrm{CE}\left(\lambda_{2}\right)$ of $\mathrm{GC}_{3}$ is optimized to obtain a $\mathrm{GC}_{4}$ with a modified direct-search algorithm [66]. As shown in Figs. 5(c) and 5(d), two prototype focusing DWB SWGCs were experimentally measured where $\operatorname{CE}(1486.0 \mathrm{~nm}, 1594.5 \mathrm{~nm})=$ $(18.3 \%, 20.1 \%)$ and $\mathrm{CE}(1481.5 \mathrm{~nm}, 1661.5 \mathrm{~nm})=(14.5 \%$, $17.5 \%)$ with the $3-\mathrm{dB}$ bandwidth of $(55.0,30.5) \mathrm{nm}$ and (44.0, $>39.5) \mathrm{nm}$, respectively. Due to the use of an unoptimized inverse taper, there is an extra loss of $\sim 1.6 \mathrm{~dB}$ in $\mathrm{CE}$. Numerically predicted $\Delta \lambda$ of $\mathrm{GC}_{4}$ covers a range of $50-350 \mathrm{~nm}$ when $\mathrm{CE}>30 \%$. Therefore, the need for exceptionally broadband GCs, which are normally limited by the trade-off between the $\mathrm{CE}$ and 1-dB bandwidth, can be bypassed. The DWB SWGCs facilitate direct coupling of widely separated wavelength bands in systems such as wavelength-divisionmultiplexing communications $[82,85]$ and the pump and Stokes wavelengths in integrated Raman amplifiers or integrated Raman spectrometers [110, 111].

\section{E. Mid-IR SWGC}

In 2012, a fully etched SWGC was demonstrated on a Sion-sapphire wafer for the TM-mode operation with a CE of $11.6 \%$ at the wavelength of $2.75 \mu \mathrm{m}$ [112]. CEs of $24.7 \%$ and $28.2 \%$ at $2.75 \mu \mathrm{m}$ were measured based the air-hole and fishbone-like suspended SWGCs, respectively [49, 50]. J. Kang and co-workers demonstrated 2D uniform focusing SWGCs on a suspended-membrane germanium $(\mathrm{Ge})$ waveguide platform 
in 2017 [113]. The coupling strength is $0.13 \mu \mathrm{m}^{-1}$ with a measured $\mathrm{CE}$ of $-11 \mathrm{~dB}$ and a $1-\mathrm{dB}$ bandwidth of $\sim 58 \mathrm{~nm}$ at the wavelength of $2.37 \mu \mathrm{m}$. The above studies are all based on the uniform GCs. The first implementation of a linearly apodized GC working at $\lambda$ of $5.2 \mu \mathrm{m}$ was demonstrated with one of the highest CEs of $-4 \mathrm{~dB}$ by S. Radosavljevic et al. in 2017 [114].

\section{SUBWAVELENGTH-STRUCTURED FULLY SUSPENDED WAVEGUIDE PLATFORMS}

Operation in the mid-IR region brings important new potential applications to $\mathrm{Si}$ photonics [115]. A challenge in moving to longer wavelengths comes from the absorption of the buried oxide in conventional SOI based silicon waveguides. The spectral ranges of transparency of $\mathrm{Si}$ and $\mathrm{SiO}_{2}$ cover 1.28.0 and $0.5-2.3 \mu \mathrm{m}$, respectively, while it is $2-15 \mu \mathrm{m}$ in $\mathrm{Ge}$ [116]. By locally etching away the BOX, air-cladding Si and Ge waveguides are promising candidates [117]. In 2012, our group first demonstrated a $\mathrm{Si}$ suspended-membrane waveguide (SMW) platform for mid-IR wavelengths based on the bilayer fabrication steps, in which the rib waveguide core was shallowly etched and two arrays of periodic air holes were deeply etched to facilitate removing of the BOX [52]. Low dispersion (within $\pm 100 \mathrm{ps} / \mathrm{nm} / \mathrm{km}$ ) was theoretically predicted within a large bandwidth of $1500 \mathrm{~nm}$. Nonlinear coefficient of a SMW was numerically estimated to be $5 \mathrm{~W}^{-1} \mathrm{~m}^{-1}$. The propagation loss was measured as $3.0 \pm 0.7 \mathrm{~dB} / \mathrm{cm}$ at the wavelength of $2.75 \mu \mathrm{m}$. Also, the SMW ring resonator was experimentally characterized exhibiting a loaded $Q$ factor of 8,100 . The SMW platform is promising for nonlinear optics [118]. With a monolayer graphene on top, long-range lightgraphene interactions enable broadband light detection with a responsivity of $0.13 \mathrm{~A} / \mathrm{W}$ at the wavelength of $2.75 \mu \mathrm{m}$ [119], nonlinear absorption [120], and all-optical modulation [121]. SMWs were also exploited on a Ge-on-insulator $(\mathrm{GeOI})$ wafer [122]. Since 2017, a set of photonic devices including focusing SWGCs [113], high- $Q$ ring resonators [123], hexagonal-lattice zerocell $\mathrm{PhC}$ cavities [124], and high- $Q$ nanobeam cavities [125] were experimentally demonstrated on the Ge SMW platform. Another novel approach is using metamaterial SWG to support an air-cladding strip waveguide [126]. Working in the subwavelength region, diffraction effects are avoided. It requires only a single lithography and etching step. Measured propagation loss was improved from 3.4 to $0.8 \mathrm{~dB} / \mathrm{cm}$ at the wavelength of $3.8 \mu \mathrm{m}$ by J. Soler Penadés et al. [127]. The bending loss of a $90^{\circ}$ bend is $0.014 \mathrm{~dB}$. In 2018, a suspended strip waveguide was demonstrated at the wavelength of $7.67 \mu \mathrm{m}$ with a low propagation loss of $3.1 \pm 0.3 \mathrm{~dB} / \mathrm{cm}$ [128]. The guided modes are mainly confined to be waveguide cores of the SMW and suspended strip waveguide, with weak overlap between the evanescent fields and the ambient. In spectroscopic and sensing applications, slot waveguides featuring direct interfacing could be an excellent candidate [129]. In the mid-IR spectral region, $\mathrm{Si}$ slot waveguides sitting on $\mathrm{BOX}$ were demonstrated with a low propagation loss of $1.4 \pm 0.2 \mathrm{~dB} / \mathrm{cm}$ [130]. A propagation loss of $11 \mathrm{~dB} / \mathrm{cm}$ was measured for a $\mathrm{Si}$ slot waveguide sitting on sapphire [131].

\section{A. Fully suspended slot waveguides (FSSWs)}

Aimed at enhancing the optical mode overlap with the analyte in integrated optical gas and microfluidic sensors, we introduced the subwavelength-structured FSSW in 2017 [53]. In 2018, we extended this approach to a full platform, including directional couplers, microring resonators, and mode convertors operating in the mid-IR spectral region [54]. The subwavelength-structured FSSW platform offers the following advantages: (1) A broad spectral range of transparency (2-8 $\mu \mathrm{m})$ is readily available, limited only by the absorption of $\mathrm{Si}$. (2) Direct light-analyte interfacing is accessible with a significant portion of power (as large as $42.3 \%$ ) in the analyte. (3) Anchored by the SWGs, the FSSW platform has good mechanical stability without structural deformation. (4) The single-layer fabrication process is simple and robust. (5) FSSWs are scalable and can be implemented at other wavelengths or on other material systems.

In a FSSW, the metamaterial SWGs can be treated as an inplane waveguide cladding based on EMT [28], and light propagates in a FSSW without the diffraction effects when the subwavelength condition is satisfied. For example, designed on a $340-\mathrm{nm}$ SOI and at $\lambda$ of $2250 \mathrm{~nm}$, the Bragg grating period $\Lambda_{\mathrm{B}}$ is $630 \mathrm{~nm}$ when $\left(W_{\mathrm{WG}}, W_{\text {slot }}, L_{\mathrm{Si}}, \Lambda, \Delta W\right)=(800,70,120,450$, $0) \mathrm{nm}$. These defined dimensions are shown in Figs. 6(a) and 6(d). Note that, even though light at a short wavelength may experience the Bragg reflection, one can decrease either $\Lambda$ or $n_{\text {eff }}$ according to $\lambda_{\text {Bragg }}=2 n_{\text {eff }} \Lambda$ to blue-shift $\lambda_{\text {Bragg, }}$ which is the center wavelength of the PBG. Figure 6 shows SEM images of the symmetric and asymmetric FSSWs. To test mechanical stability, we cleaved FSSWs and examined their cross sections. The narrow slots are etched through, and two separated waveguide cores anchored by the lateral rigid SWGs are maintained on a same plane without vertical offset as shown in Figs. 6(b), 6(c), 6(e) and 6(f). According to the FEM calculation [104], the maximal deformation of the FSSWs is only 3.6 times larger than that of the corresponding suspended strip waveguide
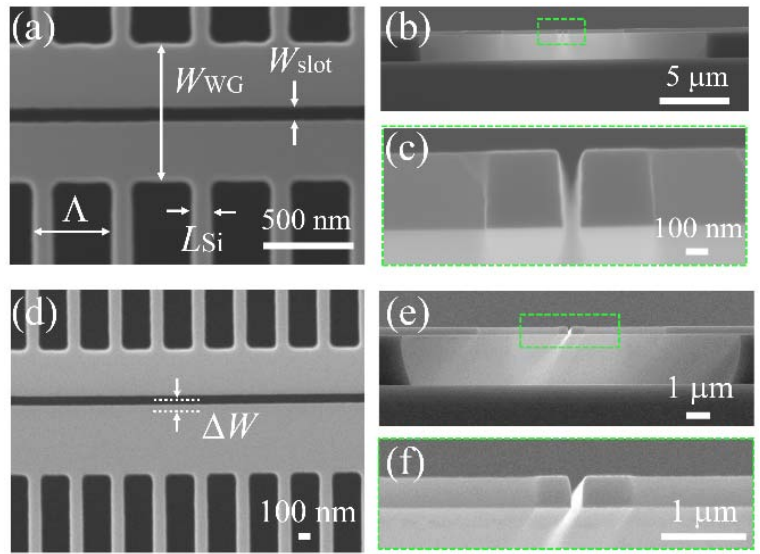

Fig. 6. SEM images of the fabricated symmetric and asymmetric FSSWs. (a) Top-view SEM image of a symmetric FSSW with dimension labels. (b, c) Cross-sectional view (b) and zoom-in (c) of a cleaved waveguide end of a symmetric FSSW. (d) Top-view of an asymmetric FSSW with a dimension label. (e, f) Cross-sectional view (e) and zoom-in (f) of a cleaved waveguide end of an asymmetric FSSW. Figures are reproduced from: (a-c) Ref. [53] with the permission of OSA; (d-f) Ref. [54] with the permission of AIP Publishing. 
without slot under the same applied pressure.

The propagation loss of the symmetric and asymmetric FSSWs with ( $\left.W_{\mathrm{WG}}, W_{\text {slot }}, L_{\mathrm{Si}}, \Lambda, \Delta W\right)$ of $(879,81,110,467,0)$ and $(1048,75,103,350,97) \mathrm{nm}$ were characterized using the linear regression method [54], which are $7.9 \mathrm{~dB} / \mathrm{cm}$ and 2.8 $\mathrm{dB} / \mathrm{cm}$ at $2.25 \mu \mathrm{m}$, respectively. There are two sources contributing to the propagation loss: (i) scattering at rough sidewalls and (ii) modal mismatch at waveguide stitches. The asymmetric FSSW has a relatively low loss due to a small fraction of intensity field overlapping with the sidewalls (3.4\%) and a small modal mismatch $(0.3 \%)$ at waveguide stitches with a 30-nm offset. The measured and fitted bending loss increases from 0.28 to $0.42 \mathrm{~dB} / 90^{\circ}$ within a spectral range of 2.14-2.31 $\mu \mathrm{m}$ for a symmetric FSSW with a bend radius $\left(R_{\text {bend }}\right)$ of 23.4 $\mu \mathrm{m}$. The bending loss is reduced to $0.15 \mathrm{~dB} / 90^{\circ}$ with $\Delta W=104$ $\mathrm{nm}$ and $R_{\text {bend }}=23.4 \mu \mathrm{m}$. The maximal Si absorption loss in the FSSWs is predicted to be $\sim 1.9 \mathrm{~dB} / \mathrm{cm}$ in the wavelength range of 2-8 $\mu \mathrm{m}$. In contrast, the absorption loss is greater than 3 $\mathrm{dB} / \mathrm{cm}$ at $\lambda>5 \mu \mathrm{m}$, and increases to $210 \mathrm{~dB} / \mathrm{cm}$ at $\lambda=8 \mu \mathrm{m}$ for the conventional slot-on-BOX platform. Due to strong enhancement of the evanescent field in the waveguide slot region, RI sensitivity could be larger than that of a free-space beam (RI sensitivity $=1 \mathrm{RIU} / \mathrm{RIU}$, where RIU stands for refractive index unit) $[132,133]$. The predicted RI sensitivity of a FSSW is $1.1 \mathrm{RIU} / \mathrm{RIU}$, which has $9.7 \%$ improvement compared with the maximal value of the slot-on-BOX waveguides, due to significant portions of optical power $(42.3 \%)$ and intensity $(87.2 \%)$ located in the ambient.

\section{B. Fully suspended waveguide devices}

To bridge the FSSW and suspended strip waveguide, a highly efficient, broadband, and compact strip-to-slot mode converter was demonstrated by our group. According to FEM simulation, the effective-index difference between the strip and (a)

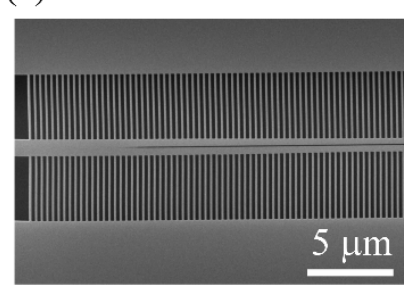

(c)

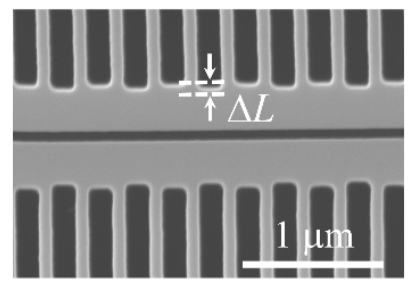

(b)

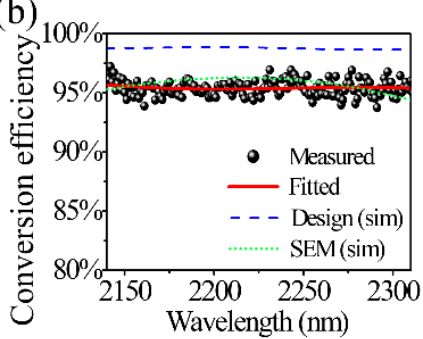

(d)

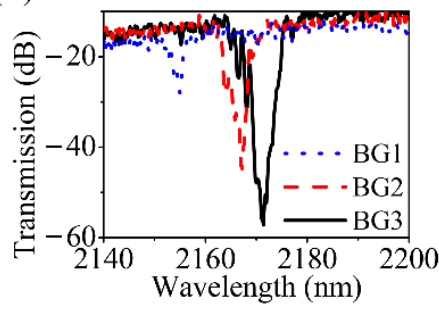

Fig. 7. SEM images and characterization of the fabricated FSSW devices. (a) Top-view SEM image of a strip-to-slot mode converter. (b) Measured and simulated conversion-efficiency spectra. (c) Top view of a sidewall Bragg grating. (d) Measured transmission spectra of three Bragg gratings with different width difference $\Delta L$. Figures are reproduced from: (a, b) Ref. [54] with the permission of AIP Publishing.
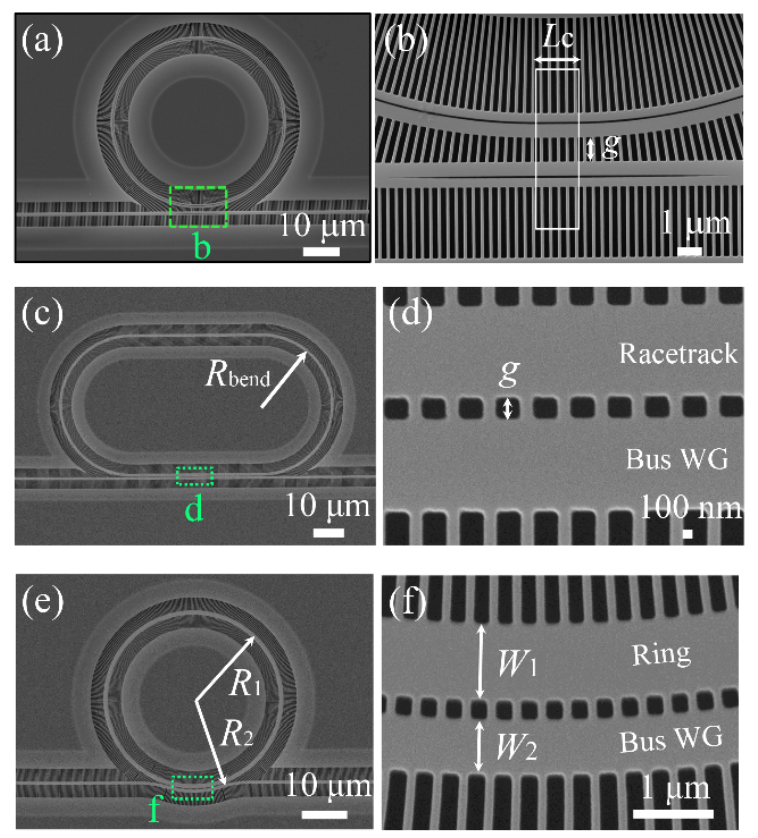

Fig. 8. SEM images of the fabricated microresonators with metamaterial SWG claddings. (a, b) Top-view SEM images of a racetrack resonator based on an asymmetric FSSW (a) and close-up of its coupling region (b). (c, d) SEM images of a racetrack resonator based on a suspended strip waveguide (c) and close-up of its coupling region based on a symmetric directional coupler (d). $(e, f)$ SEM images of a ring resonator based on a suspended strip waveguide (e) and close-up of its coupling region based on an asymmetric bent coupler (f). Figures are reproduced from: $(a, b)$ Ref. [54] with the permission of AIP Publishing; (c-f) Ref. [57].

slot waveguides is 0.87 . The average conversion efficiency can be improved from $72.9 \%$ to $98.7 \%$ with the help of an inverse slot taper. A top-view SEM image of a fabricated converter with a device length of $20 \mu \mathrm{m}$ is shown in Fig. 7(a). The measured conversion efficiency is $97.0 \%$ at $2231 \mathrm{~nm}$. The fitted and simulated spectra match well, which all have nearly flat spectral response over a 170-nm bandwidth as shown in Fig. 7(b). Sidewall Bragg gratings (BGs) were exploited on the FSSW platform. Figure 7(c) shows a SEM image of a fabricated BG. The outer sidewall of a FSSW is corrugated with a width difference $\Delta L$ in the neighboring unit cells to tune the coupling coefficient $\kappa$, which is calculated from $\pi n_{\mathrm{g}} \Delta \lambda / \lambda_{0}^{2}$ where $n_{\mathrm{g}}, \Delta \lambda$, and $\lambda_{0}$ are the group index, bandwidth, and center wavelength, respectively [134]. $\Delta L$ is $10 \mathrm{~nm}, 20 \mathrm{~nm}$, and $30 \mathrm{~nm}$ in BG1-3, respectively. The length of all BGs is $480 \mu \mathrm{m}$. With sidewall corrugation, light at the Bragg-resonance wavelength is reflected back as shown in Fig. $7(\mathrm{~d})$. The measured $\left(\lambda_{\text {Bragg, }}\right.$ ER, $\kappa)$ in units of $\left(\mathrm{nm}, \mathrm{dB}, \mathrm{m}^{-1}\right)$ of BG1-3 are $(2155.0,12.7,0.9 \times$ $\left.10^{4}\right),\left(2167.2,32.6,1.9 \times 10^{4}\right)$, and $\left(2171.4,45.7,2.1 \times 10^{4}\right)$, respectively, where ER stands for the extinction ratio.

Fully suspended slot and strip waveguide ring and racetrack resonators with metamaterial SWG claddings were demonstrated with high loaded $Q$ factors and tailorable operating bandwidths $[54,56,57]$. Figure 8 (a) shows a topview SEM image of an asymmetric FSSW racetrack resonator, and Fig. 8(b) zooms in at its coupling region. Figures 8(c) and 8(e) show SEM images of racetrack and ring resonators based on the suspended strip waveguides, and Figs. 8(d) and 8(f) 

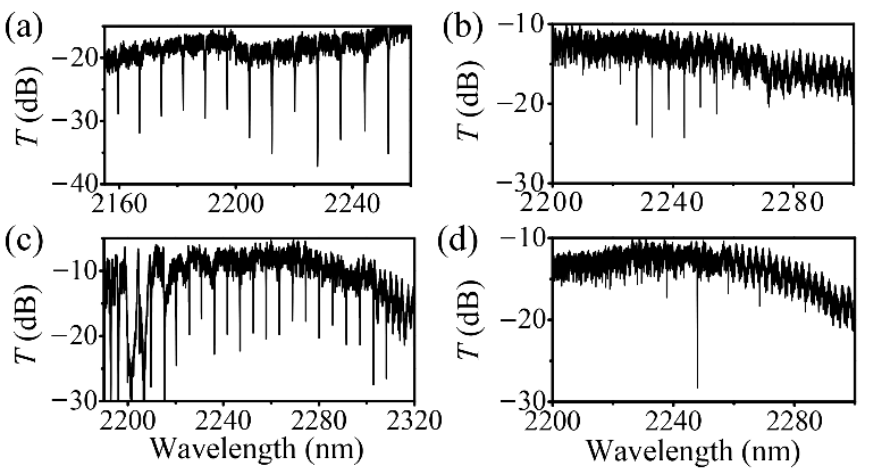

Fig. 9. Measurements of the fabricated microresonators with metamaterial SWG claddings. (a-d) Transmission spectra of a racetrack resonator based on an asymmetric FSSW (a), two racetrack resonators based on the suspended strip waveguides with $\Lambda$ of $0.36 \mu \mathrm{m}$ (b) and $0.42 \mu \mathrm{m}$ (c), a ring resonator based on a suspended strip waveguide with an asymmetric bent coupler structure (d). Figures are reproduced from: (a) Ref. [54] with the permission of AIP Publishing; (b-d) Ref. [57].

zoom in at their coupling regions, respectively. The loaded $Q$ factor of a symmetric FSSW racetrack is 1,650 at the wavelength of $2326.2 \mathrm{~nm}$ [55]. The loaded and intrinsic $Q$ factors of an asymmetric FSSW racetrack are 8,558 and 12,600 respectively at the wavelength of $2159.7 \mathrm{~nm}$ with ( $W_{\mathrm{WG}}, W_{\text {slot }}$, $\left.L_{\mathrm{Si}}, \Lambda, \Delta W, g, R_{\text {bend }}\right)=(0.94,0.08,0.12,0.35,0.1,1.0,23.4) \mu \mathrm{m}$. ER reaches $20 \mathrm{~dB}$ by fine tuning the coupling coefficient as shown in Fig. 9(a). The maximal loaded $Q$ factor is 40,600 for the suspended strip waveguide racetrack resonators. We observed Fano-like resonances during spectral scanning with $\mathrm{mW}$-level light injection due to moisture and surface states from a sample tested in the ambient. In a strip waveguide racetrack resonator with $\left(W_{\mathrm{WG}}, L_{\mathrm{Si}}, g, R_{\text {bend }}\right)=(0.91,0.11,0.22$, 23.9) $\mu \mathrm{m}$, the operating bandwidth, which is defined here as the wavelength range during which the ER is larger than $4 \mathrm{~dB}$, can be enlarged from a normal value of $43 \mathrm{~nm}$ as shown in Fig. 9(b) to $93 \mathrm{~nm}$ as shown in Fig. 9(c), by only enlarging $\Lambda$ from 0.36 to $0.42 \mu \mathrm{m}$. With the presence of the PBGs located at $\sim 2.2 \mu \mathrm{m}$ as shown in Fig. 9(c), the wavelength-dependent coupling coefficient $\kappa(\lambda)$ can be flattened, which is simulated to be between 0.07 and 0.09 in the wavelength range of 2208-2350 $\mathrm{nm}$, to match the round-trip-loss coefficient [41]. There are two PBGs because the periods of the inner and outer SWGs are slightly different. As shown in Fig. 9(d), in the reverse way, excitation of fewer modes was realized in a ring with $\left(W_{1}, W_{2}\right.$, $\left.\Lambda, L_{\mathrm{Si}}, g, R_{1}, R_{2}\right)=(0.95,0.69,0.30,0.11,0.23,20.0,21.1) \mu \mathrm{m}$. There is only a prominent resonance at the wavelength of $2247.9 \mathrm{~nm}$ with ER of $15.7 \mathrm{~dB}$, which is at least $10 \mathrm{~dB}$ larger than those of the sideband resonances. With an asymmetric bent coupler, dispersion of $\kappa(\lambda)$ can be enhanced, which is proportional to the group index difference between the even and odd supermodes [135]. According to our FDTD calculation [136], the group-index difference can be increased by $\sim 3$ times in an asymmetric bent coupler compared with that in a symmetric directional coupler. The broadband and narrowband resonances in the suspended strip waveguide resonators can benefit applications in absorption spectroscopy and FSR-free RI sensing, respectively.

\section{HyPerUNiform Disordered Photonic Structured WAVEGUIDE POLARIZERS}

The concept of hyperuniformity describes point pattern distributions, whose number variance $\sigma^{2}=\left\langle N^{2}(R)\right\rangle-\langle N(R)\rangle^{2}$ $[N(R)$ : the number of points inside a sampling window with a radius $R$ and an arbitrary location in the point pattern] grows more slowly than the window volume $(V)$ in $3 \mathrm{D}$ or window area $(A)$ in 2D with increasing $R$ [67]. Hyperuniform point pattern follows $\sigma^{2} \sim R$, unlike completely random point distributions, e. g., Poisson point pattern, in which the number variance follows $\sigma^{2} \sim R^{2}$. HUDPS is a new class of PBG material. Its research started from 2009 with discovery of the optical properties of HUDPS by M. Florescu et al. [32]. Compared with the conventional periodic lattice PhCs, HUDPS has the following six characteristics. First, a HUDPS created by the hyperuniform disordered point and network patterns supports isotropic and complete PBGs, which were demonstrated in the microwave region by W. Man et al. [137]. Freeform wave guiding and bending with an arbitrary polarization state can be realized. Second, HUDPS supports sizeable PBGs. The normalized bandwidth of a PBG is defined as the gap-tomidgap ratio $\Delta \omega^{\prime} \omega_{0}$, where $\Delta \omega$ and $\omega_{0}$ are respectively the frequency range and center frequency of a PBG. For example, $\Delta \omega / \omega_{0}$ is 0.37 and 0.34 for the TM and TE modes, respectively, in the HUDPS with a stealthiness parameter $\chi=0.5$ [32]. Third, the isotropic hyperuniformity can be implemented into a 3D structure. High-quality 3D network HUDPS exhibiting pronounced PBGs were fabricated by N. Muller et al. with direct laser writing [138]. Fourth, spatially localized modes with high $Q$ factors are supported at the band edge of a HUDPS, based on which $\mathrm{H}$. Noh et al. demonstrated an amorphous network laser based on a GaAs membrane embedded with InAs quantum dots [60]. R. Degl'Innocenti et al. demonstrated a terahertz quantum cascade laser based on a pillar-array HUDPS with unconventional emission patterns [139]. Fifth, based on the diffusion and multiple scattering, the wavelength-dependent speckle patterns in a HUDPS were leveraged to develop a compact integrated spectrometer with a spectral resolution of $0.75 \mathrm{~nm}$ [140]. Sixth, with a long diffusion length, the integrated absorption efficiency was enhanced by $5 \%$ in an amorphously patterned thin-film solar cell compared with that of the periodically patterned counterparts [61]. These theoretical and experimental studies show great potential of applying HUDPS in 2D and 3D photonic integration. $\mathrm{M}$. Milošević et al. demonstrated 2D line-defect waveguides and point-defect all-pass filters in HUDPS based on a 220-nm SOI with a measured propagation loss of $13 \mathrm{~dB} / \mathrm{cm}$ and a thermal shift rate of $\sim 0.15 \mathrm{pm} / \mathrm{K}$, respectively [141].

Based on the collective coordinate approach [142], we generated HUDS point patterns with point numbers of 56, 125, and 250 as shown in Figs. 10(a1), 10(a2), and 10(a3), respectively. These point patterns can be classified into the stealthy-type HUDS as validated from their structure factors as shown in Figs. 10(b1), 10(b2), and 10(b3). In each structure factor, there is a central disk with a radius of $|k|<k_{\mathrm{c}}$, in which $S\left(k_{x}, k_{y}\right)$ is mathematically constrained to be zero, except $S(0,0)$ 

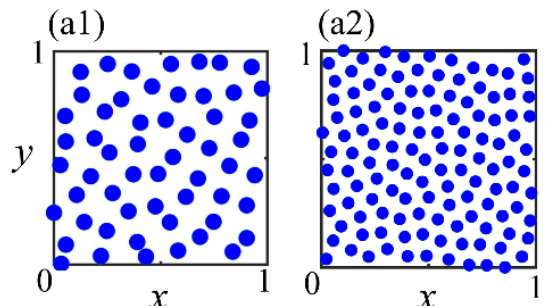

(a3)
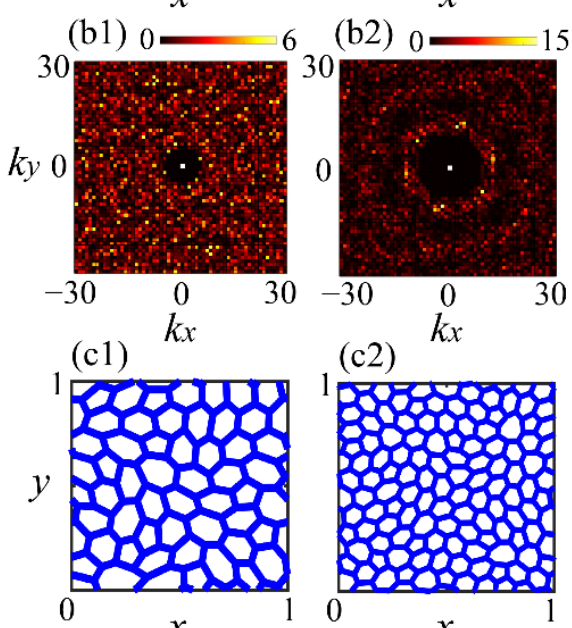

(c2)

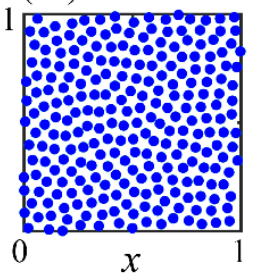

(b3)

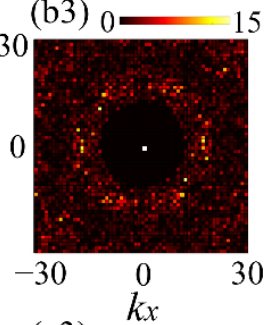

(c3)

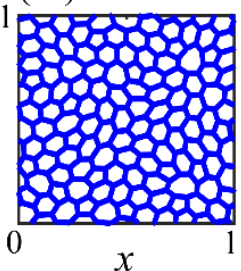

(a)

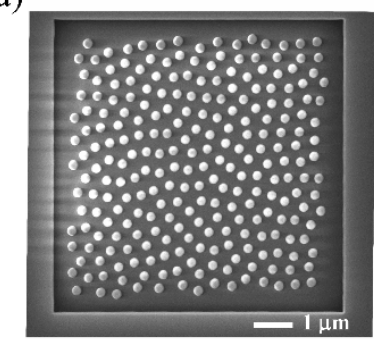

(c)

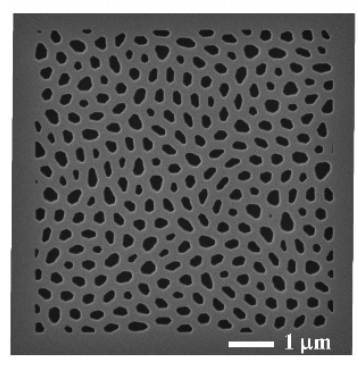

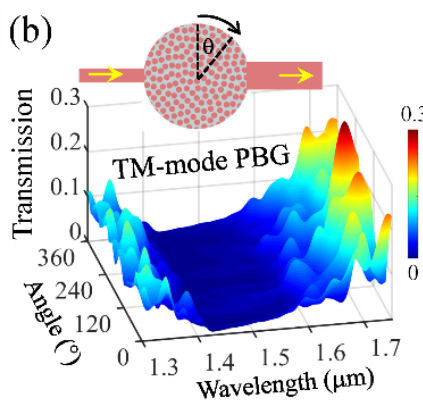

(d)

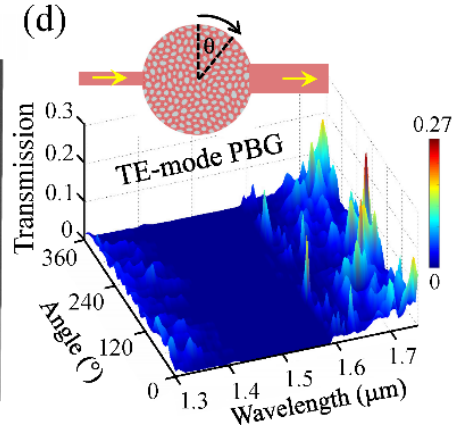

Fig. 11. Fabrication and simulated transmission spectra of Si HUDPS. (a) SEM image of a fabricated Si HUDPS based on a point pattern with 250 points. (b) Angular transmission spectra for the TM mode of a disk-shaped Si HUDPS as shown in the inset. (c) SEM image of a fabricated Si HUDPS based on a converted network pattern. (d) Angular transmission spectra for the TE mode of a disk-shaped Si HUDPS as shown in the inset. Figures are reproduced from: (a, c, d) Ref. [69] with the permission of IEEE. patterns with 56 points (a1), 125 points (a2), and 250 points (a3). (b1-b3) Structure factors of the HUDS point patterns with 56 points (b1), 125 points (b2), and 250 points (b3). (c1-c3) Converted wall-network patterns from the HUDS point patterns with 56 points (c1), 125 points (c2), and 250 points (c3).

$=N$, where $N$ is the total number of points. Outside the central disk, $S\left(k_{x}, k_{y}\right)$ has wild values without any constrain. The stealthiness parameter $\chi$ defined by $M(k) / 2 N$, where $M(k)$ is the number of constrained $k$ components inside the central disk, is $0.4,0.5$, and 0.48 for the three patterns with 56,125 , and 250 points, respectively. By applying the Delaunay triangulation method, HUDS point patterns were converted into HUDS wallnetwork patterns as shown in Figs. 10(c1), 10(c2), and 10(c3) [32]. The isotropic TM (TE)-mode PBG can be designed with a HUDPS when a HUDS point (network) pattern is decorated with dielectric rods (walls). The isotropic and complete PBG can be formed in a composite HUDPS with combined HUDS point and wall-network patterns [32, 137]. Size and location of a PBG can be tailorable by varying the average period, radius of rods, width of wall networks, and RIs of the core and cladding materials for a HUDPS.

SEM images of fabricated Si HUDPS based on the point and network patterns are respectively shown in Figs. 11(a) and 11(c). Next, isotropic PBGs for the TM and TE modes are numerically investigated with angular transmission spectra of the disk-shaped Si HUDPS as shown in the insets of Figs. 11(b) and $11(\mathrm{~d})$, respectively. In 3D FDTD simulation, Si HUDPS is placed on BOX with air superstrate and is rotated by one round with a step of $20^{\circ}$. Widths of the input and output waveguides are 1 and $2 \mu \mathrm{m}$, respectively. As shown in Fig. 11(b), a nearly isotropic TM-mode PBG covers 1419 to $1562 \mathrm{~nm}$ in wavelength, where the transmission is less than $1 \%$, in a HUDPS based on a Si rod array with an average period $\left(P_{\text {ave }}\right)$ of $333.0 \mathrm{~nm}$, radius of $130 \mathrm{~nm}$, and Si thickness of $1.5 \mu \mathrm{m}$. As

shown in Fig. 11(d), a nearly isotropic TE-mode PBG covers 1423 to $1588 \mathrm{~nm}$ in wavelength, where the transmission is less than $0.3 \%$, in a HUDPS based on a Si wall network with a $P_{\text {ave }}$ of $378.4 \mathrm{~nm}$, wall width of $185 \mathrm{~nm}$, and Si thickness of 0.34 $\mu \mathrm{m}$.

The PBG for the TE mode produced by the Si network HUDPS was used in a compact polarizer, which exploited the PBG to block the TE mode but allow the TM mode to pass in a waveguide polarizer [69]. The average period of the HUDPS is at the wavelength scale for the TE mode, which is blocked by the Bragg reflection and out-of-plane scattering, but HUDPS is a subwavelength structure for transmission of the TM mode. The etch angle is a critical parameter in the fabrication of the network HUDPS polarizer. 3D FDTD simulation showed that the bandwidths of HUDPS polarizers with vertical and $4^{\circ}$ offvertical sidewalls are respectively $91 \mathrm{~nm}$ and $110 \mathrm{~nm}$ when the ER is larger than $30 \mathrm{~dB}$. When the off-vertical angle of sidewalls is larger than $13^{\circ}$, the maximal ER cannot reach 30 dB. According to SEM characterization, the network HUDPS polarizer shown in the inset of Fig. 12 had an etch angle of $4^{\circ}$. Length and width of the HUDPS are 4.0 and $0.8 \mu \mathrm{m}$, respectively. Figure 12 shows the measured bandwidth of 98 $\mathrm{nm}(1452-1550 \mathrm{~nm})$ when the ER is larger than $30 \mathrm{~dB}$, which is one of the largest bandwidths to our knowledge for SOI waveguide polarizers. The average insertion loss of the TM mode is $1.72 \mathrm{~dB}$. Interestingly, the adverse scattering observed in light propagation and resonance [141] was used to reduce the average back reflection to $81.8 \%$ in a network HUDPS polarizer. The HUDPS polarizer is fabrication tolerant on etched widths, with well-maintained performances with an 


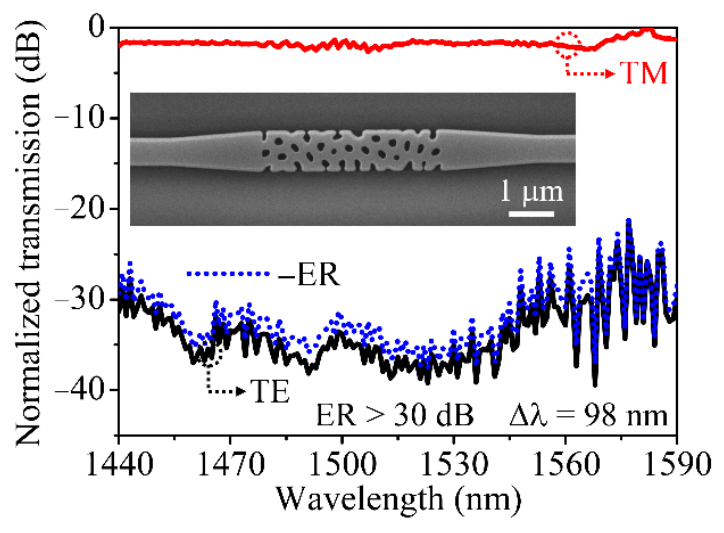

Fig. 12. Measured normalized transmission spectra for the TE and TM modes respectively indicated by the black and red solid curves. The ER between the TE and TM modes is shown as the blue dotted curve. The inset shows a SEM image of a Si network HUDPS polarizer. Figure is reproduced from Ref. [69] with the permission of IEEE.

acceptable width variation between -25 and $15 \mathrm{~nm}$. This work shows improved performances compared with those of the periodic SWG- and PhC-based polarizers [70, 71], by utilizing the HUDPS with controlled disorder. The use of HUDPS for waveguide polarizers may outperform the periodic counterparts in subwavelength engineering, and have more relaxed fabrication tolerance requirements.

\section{CONCLUSION}

We reviewed recent work on subwavelength-structured photonic integrated devices for near- and mid-IR applications. SWGs are an important tool for device engineers in enabling the engineering of a continuous range of RI, with a control of the birefringence and dispersion of the metamaterial. We have covered the use of SWG in the engineering of GCs with efficient coupling, broad bandwidth, polarization insensitivity, and dual-wavelength-band coupling. We also reviewed the fully suspended waveguide platforms for low-loss and broadband operation in the mid-IR region, and HUDPS polarizers with overall improved performances. By combining the merits of conventional Si photonics with the metamaterial SWGs and PBG-material HUDPS, it will be possible to engineer passive functional components with improved and unprecedented performances.

\section{REFERENCES}

[1] D. Thomson et al., "Roadmap on silicon photonics," J. Opt., vol. 18, no. 7, pp. 073003, Jul. 2016.

[2] A. Lim et al., "Review of silicon photonics foundry efforts," IEEE J. Sel. Top. Quantum Electron., vol. 20, no. 4, pp. 8300112, Jul. 2014.

[3] Y. Li, Y. Zhang, L. Zhang, and A. W. Poon, "Silicon and hybrid silicon photonic devices for intra-datacenter applications: state of the art and perspectives," Photonics Res., vol. 3, no. 5, pp. B10-B27, Oct. 2015.

[4] K. Xu et al., "Compatibility of silicon Mach-Zehnder modulators for advanced modulation formats," J. Lightwave Technol., vol. 31, no. 15, pp. 2550-2554, Aug. 2013.

[5] X. Wu, C. Huang, K. Xu, C. Shu, and H. K. Tsang, "128-Gb/s line rate OFDM signal modulation using an integrated silicon microring modulator," IEEE Photonics Technol. Lett., vol. 28, no. 19, pp. 20582061, Oct. 2016.
[6] P. Dong, "Silicon photonic integrated circuits for wavelength-division multiplexing applications," IEEE J. Sel. Top. Quantum Electron., vol. 22, no. 6, pp. 6100609, Nov. 2016.

[7] J. Du et al., "High speed and small footprint silicon micro-ring modulator assembly for space-division-multiplexed 100-Gbps optical interconnection," Opt. Express, vol. 26, no. 11, pp. 13721-13729, May 2018.

[8] P. Dong, C. Xie, L. Chen, L. Buhl, and Y. Chen, "112-Gb/s monolithic PDM-QPSK modulator in silicon," Opt. Express, vol. 20, no. 26, pp. B624-B629, Dec. 2012.

[9] X. Wu, C. Huang, K. Xu, W. Zhou, C. Shu, and H. K. Tsang, "3 × 104 $\mathrm{Gb} / \mathrm{s}$ single- $\lambda$ interconnect of mode-division multiplexed network with a multicore fiber," J. Lightwave Technol., vol. 36, no. 2, pp. 318-324, Jan. 2018.

[10] G. T. Reed, G. Z. Mashanovich, F. Gardes, and D. Thomson, "Silicon optical modulators," Nat. Photonics, vol. 4, no. 8, pp. 518-526, Aug. 2010.

[11] A. Politi, J. Matthews, M. G. Thompson, and J. L. O'Brien, "Integrated quantum photonics," IEEE J. Sel. Top. Quantum Electron., vol. 15, no. 6, pp. 1673-1684, Nov. 2009.

[12] J. Wang et al., "Multidimensional quantum entanglement with large-scale integrated optics," Science, vol. 360, no. 6386, pp. 285, Apr. 2018.

[13] M. Kues et al., "On-chip generation of high-dimensional entangled quantum states and their coherent control," Nature, vol. 546, no. 7660, pp. 622-626, Jun. 2017.

[14] C. Reimer et al., "Generation of multiphoton entangled quantum states by means of integrated frequency combs," Science, vol. 351, no. 6278, pp. 1176-1180, Mar. 2016.

[15] L. Feng et al., "On-chip coherent conversion of photonic quantum entanglement between different degrees of freedom," Nat. Commun., vol. 7, pp. 11985, Jun. 2016

[16] L. Tombez, E. J. Zhang, J. S. Orcutt, S. Kamlapurkar, and W. M. J. Green, "Methane absorption spectroscopy on a silicon photonic chip," Optica, vol. 4, no. 11, pp. 1322-1325, Nov. 2017.

[17] A. G. Griffith et al., "Coherent mid-infrared frequency combs in siliconmicroresonators in the presence of Raman effects," Opt. Express, vol. 24, no. 12, pp. 13044-13050, Jun. 2016.

[18] X. Sun, J. Zheng, M. Poot, C. W. Wong, and H. X. Tang, "Femtogram doubly clamped nanomechanical resonators embedded in a high- $Q$ twodimensional photonic crystal nanocavity," Nano Lett., vol. 12, no. 5, pp. 2299-2305, May 2012.

[19] X. Sun, X. Zhang, M. Poot, C. Xiong, and H. X. Tang, "A superhighfrequency optoelectromechanical system based on a slotted photonic crystal cavity," Appl. Phys. Lett., vol. 101, no. 22, pp. 221116, Nov. 2012.

[20] W. Bogaerts and L. Chrostowski, "Silicon photonics circuit design: methods, tools and challenges," Laser Photonics Rev., vol. 12, no. 4, pp. 1700237, Apr. 2018.

[21] R. Halir et al., "Waveguide sub-wavelength structures: a review of principles and applications," Laser Photonics Rev., vol. 9, no. 1, pp. 2549, Jan. 2015.

[22] W. Zhou and X.-G. Huang, "Long-range air-hole assisted subwavelength waveguides," Nanotechnology, vol. 24, no. 23, pp. 235203, Jun. 2013.

[23] W. Zhou and X.-G. Huang, "Compact filters and demultiplexers based on long-range air-hole assisted subwavelength waveguides," Opt. Express, vol. 21, no. 23, pp. 28456-28468, Nov. 2013.

[24] W. Zhou, Z. Yu, J. Ma, B. Zhu, H. K. Tsang, and X. Sun, "Ultraviolet optomechanical crystal cavities with ultrasmall modal mass and high optomechanical coupling rate," Sci. Rep., vol. 6, pp. 37134, Nov. 2016.

[25] P. Cheben, R. Halir, J. H. Schmid, H. A. Atwater, and D. R. Smith, "Subwavelength integrated photonics," Nature, vol. 560, pp. 565-572, Aug. 2018.

[26] I. Staude and J. Schilling, "Metamaterial-inspired silicon nanophotonics," Nat. Photonics, vol. 11, no. 5, pp. 274-284, May 2017.

[27] M. V. Rybin, D. S. Filonov, K. B. Samusev, P. A. Belov, Y. S. Kivshar, and M. F. Limonov, "Phase diagram for the transition from photonic crystals to dielectric metamaterials," Nat. Commun., vol. 6, pp. 10102, Dec. 2015.

[28] S. M. Rytov, "Electromagnetic properties of a finely stratified medium," Sov. Phys. JETP, vol. 2, pp. 446-475, Mar. 1956.

[29] D. H. Raguin and G. M. Morris, "Antireflection structured surfaces for the infrared spectral region," Appl. Opt., vol. 32, no. 7, pp. 1154-1167, Mar. 1993.

[30] J. D. Joannopoulos, S. G. Johnson, J. N. Winn, and R. D. Meade, Photonic Crystals: Molding the Flow of Light, 2nd ed. Princeton, NJ, USA: Princeton Univ. Press, 2008. 
[31] Y. Li et al., "On-chip zero-index metamaterials," Nat. Photonics, vol. 9, no. 11, pp. 738-742, Nov. 2015.

[32] M. Florescu, S. Torquato, and P. J. Steinhardt, "Designer disordered materials with large, complete photonic band gaps," Proc. Nat. Acad. Sci. USA, vol. 106, no. 49, pp. 20658-20663, Dec. 2009.

[33] P. J. Bock et al., "Subwavelength grating periodic structures in siliconon-insulator: a new type of microphotonic waveguide," Opt. Express, vol. 18, no. 19, pp. 20251-20262, Sep. 2010.

[34] P. Cheben, D.-X. Xu, S. Janz, and A. Densmore, "Subwavelength waveguide grating for mode conversion and light coupling in integrated optics," Opt. Express, vol. 14, no. 11, pp. 4695-4702, May 2006.

[35] D. Benedikovic et al., "Dispersion control of silicon nanophotonic waveguides using sub-wavelength grating metamaterials in near- and mid-IR wavelengths," Opt. Express, vol. 25, no. 16, pp. 19468-19478, Aug. 2017.

[36] R. Halir et al., "Ultra-broadband nanophotonic beamsplitter using an anisotropic sub-wavelength metamaterial," Laser Photonics Rev., vol. 10, no. 6, pp. 1039-1046, Nov. 2016.

[37] J. M. Luque-González et al., "Tilted subwavelength gratings: controlling anisotropy in metamaterial nanophotonic waveguides," Opt. Lett., vol. 43, no. 19, pp. 4691-4694, Oct. 2018.

[38] X. Chen and H. K. Tsang, "Polarization-independent grating couplers for silicon-on-insulator nanophotonic waveguides," Opt. Lett., vol. 36, no. 6, pp. 796-798, Mar. 2011.

[39] P. J. Bock et al., "Subwavelength grating crossings for silicon wire waveguides," Opt. Express, vol. 18, no. 15, pp. 16146-16155, Jul. 2010.

[40] Z. Wang, X. Xu, D. Fan, Y. Wang, H. Subbaraman, and R. T. Chen, "Geometrical tuning art for entirely subwavelength grating waveguide based integrated photonics circuits," Sci. Rep., vol. 6, pp. 24106, May 2016.

[41] R. Halir et al., "Colorless directional coupler with dispersion engineered sub-wavelength structure," Opt. Express, vol. 20, no. 12, pp. 1347013477, Jun. 2012.

[42] P. Cheben et al., "Broadband polarization independent nanophotonic coupler for silicon waveguides with ultra-high efficiency," Opt. Express, vol. 23, no. 17, pp. 22553-22563, Aug. 2015.

[43] H. Xu and Y. Shi, "Ultra-compact polarization-independent directional couplers utilizing a subwavelength structure," Opt. Lett., vol. 42, no. 24, pp. 5202-5205, Dec. 2017.

[44] C. Li and D. Dai, "Compact polarization beam splitter for silicon photonic integrated circuits with a 340-nm-thick silicon core layer," Opt. Lett., vol. 42, no. 21, pp. 4243-4246, Nov. 2017.

[45] J. Flueckiger et al., "Sub-wavelength grating for enhanced ring resonator biosensor," Opt. Express, vol. 24, no. 14, pp. 15672-15686, Jul. 2016.

[46] X. Chen and H. K. Tsang, "Nanoholes grating couplers for coupling between silicon-on-insulator waveguides and optical fibers," IEEE Photonics J., vol. 1, no. 3, pp. 184-190, Sep. 2009.

[47] X. Chen, K. Xu, Z. Cheng, C. Fung, and H. K. Tsang, "Wideband subwavelength gratings for coupling between silicon-on-insulator waveguides and optical fibers," Opt. Lett., vol. 37, no. 17, pp. 3483-3485, Sep. 2012.

[48] Z. Cheng, X. Chen, C. Y. Wong, K. Xu, and H. K. Tsang, "Apodized focusing subwavelength grating couplers for suspended membrane waveguides," Appl. Phys. Lett., vol. 101, no. 10, pp. 101104, Sep. 2012.

[49] Z. Cheng, X. Chen, C. Y. Wong, K. Xu, and H. K. Tsang, "Broadband focusing grating couplers for suspended-membrane waveguides," Opt. Lett., vol. 37, no. 24, pp. 5181-5183, Dec. 2012.

[50] Z. Cheng et al., "Focusing subwavelength grating coupler for midinfrared suspended membrane waveguide," Opt. Lett., vol. 37, no. 7, pp. 1217-1219, Apr. 2012.

[51] Z. Cheng and H. K. Tsang, "Experimental demonstration of polarizationinsensitive air-cladding grating couplers for silicon-on-insulator waveguides," Opt. Lett., vol. 39, no. 7, pp. 2206-2209, Apr. 2014.

[52] Z. Cheng, X. Chen, C. Y. Wong, K. Xu, and H. K. Tsang, "Mid-infrared suspended membrane waveguide and ring resonator on silicon-oninsulator," IEEE Photonics J., vol. 4, no. 5, pp. 1510-1519, Oct. 2012.

[53] W. Zhou, Z. Cheng, X. Wu, B. Zhu, X. Sun, and H. K. Tsang, "Fully suspended slot waveguides for high refractive index sensitivity," Opt. Lett., vol. 42, no. 7, pp. 1245-1248, Apr. 2017.

[54] W. Zhou, Z. Cheng, X. Wu, X. Sun, and H. K. Tsang, "Fully suspended slot waveguide platform," J. Appl. Phys., vol. 123, no. 6, pp. 063103, Feb. 2018.

[55] W. Zhou, Z. Cheng, Y. Gao, X. Wu, X. Sun, and H. K. Tsang, "Fully suspended slot waveguide racetrack resonators," presented at the 19th
European Conference on Integrated Optics (ECIO), Eindhoven, The Netherlands, Apr. 3-5, 2017.

[56] W. Zhou, Z. Cheng, X. Wu, B. Zheng, X. Sun, and H. K. Tsang, "Fully suspended mid-infrared racetrack resonator with subwavelength grating cladding," presented at the IEEE 14th Group IV Photonics Conference, Berlin, Germany, Aug. 23-25, 2017.

[57] W. Zhou, Z. Cheng, X. Wu, M. Feng, X. Sun, and H. K. Tsang, "Fully suspended nanophotonic waveguide with high quality factor and tailorable operational bandwidth," presented at the OSA Asia Communication and Photonics Conference (ACP), Guangzhou, China, Nov. 10-13, 2017.

[58] D. S. Wiersma, "Disordered photonics," Nat. Photonics, vol. 7, no. 3, pp. 188-196, Mar. 2013.

[59] J. Liu et al., "Random nanolasing in the Anderson localized regime," Nat. Nanotechnol., vol. 9, no. 4, pp. 285-289, Apr. 2014.

[60] H. Noh, J.-K. Yang, S. F. Liew, M. J. Rooks, G. S. Solomon, and H. Cao, "Photonic network laser," Opt. Lett., vol. 36, no. 18, pp. 3560-3562, Sep. 2011.

[61] K. Vynck, M. Burresi, F. Riboli, and D. S. Wiersma, "Photon management in two-dimensional disordered media," Nat. Mater., vol. 11, no. 12, pp. 1017-1022, Dec. 2012.

[62] B. Shen, P. Wang, R. Polson, and R. Menon, "An integratednanophotonics polarization beamsplitter with $2.4 \times 2.4 \mu \mathrm{m}^{2}$ footprint," Nat. Photonics, vol. 9, no. 6, pp. 378-382, Jun. 2015.

[63] A. Y. Piggott, J. Lu, K. G. Lagoudakis, J. Petykiewicz, T. M. Babinec, and J. Vuckovic, "Inverse design and demonstration of a compact and broadband on-chip wavelength demultiplexer," Nat. Photonics, vol. 9, no. 6, pp. 374-377, Jun. 2015.

[64] Z. Yu, H. Cui, and X. Sun, "Genetic-algorithm-optimized wideband onchip polarization rotator with an ultrasmall footprint," Opt. Lett., vol. 42, no. 16, pp. 3093-3096, Aug. 2017.

[65] K. Xu et al., "Integrated photonic power divider with arbitrary power ratios," Opt. Lett., vol. 42, no. 4, pp. 855-858, Feb. 2017.

[66] X. Wen, K. Xu, and Q. Song, "Design of a barcode-like waveguide nanostructure for efficient chip-fiber coupling," Photonics Res., vol. 4, no. 6, pp. 209-213, Dec. 2016.

[67] S. Torquato and F. H. Stillinger, "Local density fluctuations, hyperuniformity, and order metrics," Phys. Rev. E, vol. 68, no. 4, pp. 041113, Oct. 2003.

[68] L. S. Froufe-Perez, M. Engel, J. José-Sáenz, and F. Scheffold, "Band gap formation and Anderson localization in disordered photonic materials with structural correlations," Proc. Nat. Acad. Sci. USA, vol. 114, no. 36, pp. 9570-9574, Sep. 2017.

[69] W. Zhou, Z. Cheng, B. Zhu, X. Sun, and H. K. Tsang, "Hyperuniform disordered network polarizers," IEEE J. Sel. Top. Quantum Electron., vol. 23, no. 6, pp. 4901307, Nov. 2017.

[70] Y. Cui, Q. Wu, E. Schonbrun, M. Tinker, J.-B. Lee, and W. Park, "Siliconbased 2-D slab photonic crystal TM polarizer at telecommunication wavelength," IEEE Photonics Technol. Lett., vol. 20, no. 8, pp. 641-643, Apr. 2008.

[71] X. Guan, P. Chen, S. Chen, P. Xu, Y. Shi, and D. Dai, "Low-loss ultracompact transverse-magnetic-pass polarizer with a silicon subwavelength grating waveguide," Opt. Lett., vol. 39, no. 15, pp. 45144517, Aug. 2014.

[72] D. Taillaert et al., "An out-of-plane grating coupler for efficient buttcoupling between compact planar waveguides and single-mode fibers," IEEE J. Quantum Electron., vol. 38, no. 7, pp. 949-955, Jul. 2002.

[73] R. Topley et al., "Locally erasable couplers for optical device testing in silicon on insulator," J. Lightwave Technol., vol. 32, no. 12, pp. 22482253, Jun. 2014.

[74] X. Chen et al., "Post-fabrication phase trimming of Mach-Zehnder interferometers by laser annealing of germanium implanted waveguides," Photonics Res., vol. 5, no. 6, pp. 578-582, Dec. 2017.

[75] M. Milosevic et al., "Ion implantation in silicon for trimming the operating wavelength of ring resonators," IEEE J. Sel. Top. Quantum Electron., vol. 24, no. 4, pp. 8200107, Jul. 2018.

[76] C. Li, K. Chee, J. Tao, H. Zhang, M. Yu, and G. Q. Lo, "Silicon photonics packaging with lateral fiber coupling to apodized grating coupler embedded circuit," Opt. Express, vol. 22, no. 20, pp. 24235-24240, Oct. 2014.

[77] Y. Tong, W. Zhou, and H. K. Tsang, "Efficient perfectly vertical grating coupler for multi-core fibers fabricated with $193 \mathrm{~nm}$ DUV lithography," Opt. Lett., vol. 43, no. 23, pp. 5709-5712, Dec. 2018.

[78] D. Taillaert, H. Chong, P. I. Borel, L. H. Frandsen, R. De La Rue, and R. Baets, "A compact two-dimensional grating coupler used as a polarization 
splitter," IEEE Photonics Technol. Lett., vol. 15, no. 9, pp. 1249-1251, Sep. 2003.

[79] T. Watanabe, M. Ayata, U. Koch, Y. Fedoryshyn, and J. Leuthold, "Perpendicular grating coupler based on a blazed antiback-reflection structure," J. Lightwave Technol., vol. 35, no. 21, pp. 4663-4669, Nov. 2017.

[80] X. Chen, C. Li, and H. K. Tsang, "Fabrication-tolerant waveguide chirped grating coupler for coupling to a perfectly vertical optical fiber," IEEE Photonics Technol. Lett., vol. 20, no. 23, pp. 1914-1916, Dec. 2008.

[81] G. Roelkens, D. Van Thourhout, and R. Baets, "Silicon-on-insulator ultracompact duplexer based on a diffractive grating structure," Opt. Express, vol. 15, no. 16, pp. 10091-10096, Aug. 2007.

[82] L. Xu, X. Chen, C. Li, and H. K. Tsang, "Bi-wavelength two dimensional chirped grating couplers for low cost WDM PON transceivers," Opt. Commun., vol. 284, no. 8, pp. 2242-2244, Apr. 2011.

[83] A. Y. Piggott, J. Lu, T. M. Babinec, K. G. Lagoudakis, J. Petykiewicz, and J. Vuckovic, "Inverse design and implementation of a wavelength demultiplexing grating coupler," Sci. Rep., vol. 4, pp. 7210, Nov. 2014.

[84] Y. Ding, F. Ye, C. Peucheret, H. Ou, Y. Miyamoto, and T. Morioka, "Onchip grating coupler array on the SOI platform for fan-in/fan-out of MCFs with low insertion loss and crosstalk," Opt. Express, vol. 23, no. 3, pp. 3292-3298, Feb. 2015.

[85] G. Roelkens, D. Vermeulen, S. Selvaraja, R. Halir, W. Bogaerts, and D. Van Thourhout, "Grating-based optical fiber interfaces for silicon-oninsulator photonic integrated circuits," IEEE J. Sel. Top. Quantum Electron., vol. 17, no. 3, pp. 571-580, May 2011.

[86] C. Alonso-Ramos et al., "Single-etch grating coupler for micrometric silicon rib waveguides," Opt. Lett., vol. 36, no. 14, pp. 2647-2649, Jul. 2011.

[87] A. Sanchez-Postigo et al., "Broadband fiber-chip zero-order surface grating coupler with $0.4 \mathrm{~dB}$ efficiency," Opt. Lett., vol. 41, no. 13, pp. 3013-3016, Jul. 2016.

[88] X. Chen, C. Li, C. Fung, S. Lo, and H. K. Tsang, "Apodized waveguide grating couplers for efficient coupling to optical fibers," IEEE Photonics Technol. Lett., vol. 22, no. 15, pp. 1156-1158, Aug. 2010.

[89] C. Li, H. Zhang, M. Yu, and G. Q. Lo, "CMOS-compatible high efficiency double-etched apodized waveguide grating coupler," Opt. Express, vol. 21, no. 7, pp. 7868-7874, Apr. 2013.

[90] R. Marchetti et al., "High-efficiency grating-couplers: demonstration of a new design strategy," Sci. Rep., vol. 7, pp. 16670, Nov. 2017.

[91] W. S. Zaoui et al., "Bridging the gap between optical fibers and silicon photonic integrated circuits," Opt. Express, vol. 22, no. 2, pp. 1277-1286, Jan. 2014.

[92] D. Benedikovic et al., "Subwavelength index engineered surface grating coupler with sub-decibel efficiency for 220-nm silicon-on-insulator waveguides," Opt. Express, vol. 23, no. 17, pp. 22628-22635, Aug. 2015.

[93] M. Streshinsky et al., "A compact bi-wavelength polarization splitting grating coupler fabricated in a $220 \mathrm{~nm}$ SOI platform," Opt. Express, vol. 21, no. 25, pp. 31019-31028, Dec. 2013

[94] R. Halir, P. Cheben, S. Janz, D.-X. Xu, I. Molina-Fernandez, and J. Wanguemert-Perez, "Waveguide grating coupler with subwavelength microstructures," Opt. Lett., vol. 34, no. 9, pp. 1408-1410, May 2009.

[95] J. H. Schmid, P. Cheben, S. Janz, J. Lapointe, E. Post, and D.-X. Xu, "Gradient-index antireflective subwavelength structures for planar waveguide facets," Opt. Lett., vol. 32, no. 13, pp. 1794-1796, Jul. 2007.

[96] X. Xu, H. Subbaraman, J. Covey, D. Kwong, A. Hosseini, and R. T. Chen, "Colorless grating couplers realized by interleaving dispersion engineered subwavelength structures," Opt. Lett., vol. 38, no. 18, pp. 3588-3591, Sep. 2013.

[97] F. Van Laere et al., "Compact focusing grating couplers for silicon-oninsulator integrated circuits," IEEE Photonics Technol. Lett., vol. 19, no. 23, pp. 1919-1921, Dec. 2007.

[98] D. Taillaert, P. Bienstman, and R. Baets, "Compact efficient broadband grating coupler for silicon-on-insulator waveguides," Opt. Lett., vol. 29, no. 23, pp. 2749-2751, Dec. 2004

[99] X. Chen, C. Fung, Y. Chen, and H. K. Tsang, "Subwavelength waveguide grating coupler for fiber-to-chip coupling on SOI with $80 \mathrm{~nm} 1 \mathrm{~dB}$ bandwidth," presented at the Conference on Lasers and Electro-Optics (CLEO), Maryland, USA, May 1-6, 2011.

[100]Y. Wang et al., "Design of broadband subwavelength grating couplers with low back reflection," Opt. Lett., vol. 40, no. 20, pp. 4647-4650, Oct. 2015.

[101]Z. Xiao, F. Luan, T.-Y. Liow, J. Zhang, and P. Shum, "Design for broadband high-efficiency grating couplers," Opt. Lett., vol. 37, no. 4, pp. 530-532, Feb. 2012.
[102]Z. Xiao, T.-Y. Liow, J. Zhang, P. Shum, and F. Luan, "Bandwidth analysis of waveguide grating coupler," Opt. Express, vol. 21, no. 5, pp. 56885700, Mar. 2013

[103]Z. Cheng, Z. Li, K. Xu, and H. K. Tsang, "Increase of the grating coupler bandwidth with a graphene overlay," Appl. Phys. Lett., vol. 104, no. 11, pp. 111109, Mar. 2014.

[104]COMSOL Group [Online]. Available: http://www.comsol.com/.

[105]C. Xiang, H. K. Tsang, Z. Cheng, and C.-K. Chan, "Increasing the grating coupler bandwidth with a high numerical-aperture fiber," presented at the IEEE 11th International Conference on Group IV Photonics (GFP), Paris, France, Aug. 27-29, 2014.

[106]Q. Zhong et al., "Focusing-curved subwavelength grating couplers for ultra-broadband silicon photonics optical interfaces," Opt. Express, vol. 22, no. 15 , pp. 18224-18231, Jul. 2014

[107] F. V. Laere, W. Bogaerts, P. Dumon, G. Roelkens, D. V. Thourhout, and R. Baets, "Focusing polarization diversity grating couplers in silicon-oninsulator," J. Lightwave Technol., vol. 27, no. 5, pp. 612-618, Mar. 2009.

[108] J. Zou, Y. Yu, and X. Zhang, "Two-dimensional grating coupler with a low polarization dependent loss of $0.25 \mathrm{~dB}$ covering the C-band," Opt. Lett., vol. 41, no. 18, pp. 4206-4209, Sep. 2016.

[109]W. Zhou, Z. Cheng, X. Sun, and H. K. Tsang, "Tailorable dualwavelength-band coupling in a transverse-electric-mode focusing subwavelength grating coupler," Opt. Lett., vol. 43, no. 12, pp. 2985-2988, Jun. 2018

[110]T. K. Liang and H. K. Tsang, "Efficient Raman amplification in siliconon-insulator waveguides," Appl. Phys. Lett., vol. 85, no. 16, pp. 33433345, Oct. 2004.

[111]H. Zhao, S. Clemmen, A. Raza, and R. Baets, "Stimulated Raman spectroscopy of analytes evanescently probed by a silicon nitride photonic integrated waveguide," Opt. Lett., vol. 43, no. 6, pp. 1403-1406, Mar. 2018

[112]Z. Cheng et al., "Mid-infrared grating couplers for silicon-on-sapphire waveguides," IEEE Photonics J., vol. 4, no. 1, pp. 104-113, Feb. 2012.

[113]J. Kang et al., "Focusing subwavelength grating coupler for mid-infrared suspended membrane germanium waveguides," Opt. Lett., vol. 42, no. 11, pp. 2094-2097, Jun. 2017.

[114]S. Radosavljevic, B. Kuyken, and G. Roelkens, "Efficient 5.2 um wavelength fiber-to-chip grating couplers for the Ge-on-Si and Ge-onSOI mid-infrared waveguide platform," Opt. Express, vol. 25, no. 16, pp. 19034-19042, Aug. 2017

[115]H. Lin et al., "Mid-infrared integrated photonics on silicon: a perspective," Nanophotonics, vol. 7, no. 2, pp. 393-420, Feb. 2018.

[116]R. Soref, "Mid-infrared photonics in silicon and germanium," Nat. Photonics, vol. 4, no. 8, pp. 495-497, Aug. 2010.

[117]R. A. Soref, S. J. Emelett, and W. R. Buchwald, "Silicon waveguided components for the long-wave infrared region," J. Opt. A, vol. 8, no. 10, pp. 840-848, Oct. 2006.

[118]Y. Zhang et al., "Enhancement of self-phase modulation induced spectral broadening in silicon suspended membrane waveguides," J. Opt., vol. 18, no. 5 , pp. 055503 , May 2016.

[119] X. Wang, Z. Cheng, K. Xu, H. K. Tsang, and J.-B. Xu, "High-responsivity graphene/silicon-heterostructure waveguide photodetectors," Nat. Photonics, vol. 7, no. 11, pp. 888-891, Nov. 2013.

[120]Z. Cheng, H. K. Tsang, K. Xu, and Z. Shi, "Spectral hole burning in silicon waveguides with a graphene layer on top," Opt. Lett., vol. 38, no. 11, pp. 1930-1932, Jun. 2013.

[121]Z. Cheng, H. K. Tsang, X. Wang, K. Xu, and J.-B. Xu, "In-plane optical absorption and free carrier absorption in graphene-on-silicon waveguides," IEEE J. Sel. Top. Quantum Electron., vol. 20, no. 1, pp. 4400106, Jan. 2014.

[122]J. Kang, M. Takenaka, and S. Takagi, "Novel Ge waveguide platform on Ge-on-insulator wafer for mid-infrared photonic integrated circuits," Opt. Express, vol. 24, no. 11, pp. 1855-1864, May 2016.

[123]T.-H. Xiao et al., "Mid-infrared high- $Q$ germanium microring resonator," Opt. Lett., vol. 43, no. 12, pp. 2885-2888, Jun. 2018.

[124]T.-H. Xiao et al., "Mid-infrared germanium photonic crystal cavity," Opt. Lett., vol. 42, no. 15, pp. 2882-2885, Aug. 2017.

[125]T.-H. Xiao et al., "High- $Q$ germanium optical nanocavity," Photonics Res., vol. 6, no. 9, pp. 925-928, Sep. 2018.

[126]J. Soler Penadés et al., "Suspended SOI waveguide with sub-wavelength grating cladding for mid-infrared," Opt. Lett., vol. 39, no. 19, pp. 56615664, Oct. 2014.

[127]J. Soler Penadés et al., "Suspended silicon mid-infrared waveguide devices with subwavelength grating metamaterial cladding," Opt. Express, vol. 24, no. 20, pp. 22908-22916, Oct. 2016. 
[128]J. Soler Penadés et al., "Suspended silicon waveguides for long-wave infrared wavelengths," Opt. Lett., vol. 43, no. 4, pp. 795-798, Feb. 2018.

[129] V. Almeida, Q. Xu, C. Barrios, and M. Lipson, "Guiding and confining light in void nanostructure," Opt. Lett., vol. 29, no. 11, pp. 1209-1211, Jun. 2004.

[130]J. Soler Penadés, A. Khokhar, M. Nedeljkovic, and G. Z. Mashanovich, "Low-loss mid-infrared SOI slot waveguides," IEEE Photonics Technol. Lett., vol. 27, no. 11, pp. 1197-1199, Jun. 2015.

[131]Y. Zou et al., "Grating-coupled silicon-on-sapphire integrated slot waveguides operating at mid-infrared wavelengths," Opt. Lett., vol. 39, no. 10, pp. 3070-3073, May 2014.

[132] G. J. Veldhuis, O. Parriaux, H. Hoekstra, and P. V. Lambeck, "Sensitivity enhancement in evanescent optical waveguide sensors," J. Lightwave Technol., vol. 18, no. 5, pp. 677-682, May 2000.

[133] Y. Shi, K. Ma, and D. Dai, "Sensitivity enhancement in Si nanophotonic waveguides used for refractive index sensing," Sensors, vol. 16, no. 3, pp. 324, Mar. 2016.

[134]X. Wang et al., "Precise control of the coupling coefficient through destructive interference in silicon waveguide Bragg gratings," Opt. Lett., vol. 39, no. 19, pp. 5519-5522, Oct. 2014.

[135]R. Aguinaldo, Y. Shen, and S. Mookherjea, "Large dispersion of silicon directional couplers obtained via wideband microring parametric characterization," IEEE Photonics Technol. Lett., vol. 24, no. 14, pp. 1242-1244, Jul. 2012.

[136]Lumerical Solutions, Inc. [Online]. Available: http://www.lumerical.com/tcad-products/fdtd/.

[137]W. Man et al., "Isotropic band gaps and freeform waveguides observed in hyperuniform disordered photonic solids," Proc. Nat. Acad. Sci. USA, vol. 110, no. 40, pp. 15886-15891, Oct. 2013.

[138]N. Muller, J. Haberko, C. Marichy, and F. Scheffold, "Silicon hyperuniform disordered photonic materials with a pronounced gap in the shortwave infrared," Adv. Opt. Mater., vol. 2, no. 2, pp. 115-119, Feb. 2014.

[139]R. Degl'Innocenti et al., "Hyperuniform disordered terahertz quantum cascade laser," Sci. Rep., vol. 6, pp. 19325, Jan. 2016.

[140]B. Redding, S. F. Liew, R. Sarma, and H. Cao, "Compact spectrometer based on a disordered photonic chip," Nat. Photonics, vol. 7, no. 9, pp. 746-751, Sep. 2013.

[141]M. Milosevic et al., "Silicon waveguides and filters in hyperuniform disordered photonic solids for the near-infrared," presented at the Optical Fiber Communications Conference and Exhibition (OFC), San Francisco, United States, Mar. 9-13, 2014.

[142]O. U. Uche, F. H. Stillinger, and S. Torquato, "Constraints on collective density variables: Two dimensions," Phys. Rev. E, vol. 70, no. 4, pp. 046122, Oct. 2004.

Wen Zhou was born in Wuhan, China. He received the M.S. degree from South China Normal University, China, in 2014. He received his Ph.D. degree in Electronic Engineering from The Chinese University of Hong Kong in 2018. $\mathrm{He}$ is currently a postdoctoral fellow supported by the Postdoctoral HubInnovation and Technology Fund (PH-ITF). He has authored and coauthored more than 38 papers in technical journals and international conferences. His current research interests include integrated optics, mid-infrared silicon photonics, and hyperunifrom disordered photonics.

Zhenzhou Cheng (S'11-M'14) received his B.S. degree in physics and M.S. degree in optics from Nankai University, China, in 2006 and 2009, respectively. He received his Ph.D. degree in Electronic Engineering from The Chinese University of Hong Kong in 2013. He was awarded the Postgraduate Research Output Award and the Young Scholar Thesis Award from The Chinese
University of Hong Kong, and the Young Scientist Award from Hong Kong Institute of Science in 2013. He joined the Department of Chemistry at The University of Tokyo as an Assistant Professor in 2015. He has authored and coauthored more than 90 papers in technical journals and international conferences. His research focuses on graphene and silicon photonics for sensing, spectroscopy, and optical communications.

Xia Chen (M'10) received the B.Eng. and Ph.D. degrees in Electronic Engineering from The Chinese University of Hong Kong, Shatin, Hong Kong, in 2006 and 2010, respectively. After graduation, he worked on the U.K. Silicon Photonics Project in the University of Surrey and the University of Southampton. In 2013, he joined Huawei Technologies Co., Ltd., as a Senior Engineer to work on photonic packaging. In 2015, he rejoined the Silicon Photonics group at the University of Southampton, Southampton, U.K., where he is currently a Senior Research Fellow at the Optoelectronics Research Centre. He has authored and coauthored more than 90 papers in technical journals and international conferences and 4 patents. He received the TSMC Outstanding Student Research Award in 2010, and the Newton International Fellowship by the Royal Society in 2011.

Ke Xu (M’15) was born in Wuhan, China. He received B. Eng. degree from Huazhong University of Science and Technology, Wuhan, China, in 2010. He obtained his Ph.D. degree from Department of Electronic Engineering, The Chinese University of Hong Kong, Shatin, Hong Kong, in 2014. He was a research visiting student in Department of Photonics, National Chiao Tung University, Taiwan in August, 2012 and a visiting research assistant at Yale University from September 2013 to January 2014. He was a recipient of Hong Kong Young Scientist Award, 2014 and IEEE Photonics Society Graduate Student Fellowship, 2013. He is currently an Associate Professor in Shenzhen Graduate School, Harbin Institute of Technology, Shenzhen, China. His research interest includes silicon photonics, optical interconnects, and midinfrared photonics.

Xiankai Sun (S'07-M'18) received the Ph.D. degree in Applied Physics from California Institute of Technology, USA, in 2010. From 2010 to 2014, he was a Postdoctoral Associate and then an Associate Research Scientist in Department of Electrical Engineering at Yale University, USA. In 2014, he joined Department of Electronic Engineering at The Chinese University of Hong Kong, Hong Kong, where he is currently an Assistant Professor. He has published more than 80 papers in journals and conference proceedings. He also received the 2015 Early Career Award by the Research Grants Council of Hong Kong. He is currently an Associate Editor of Optics Express and an Editorial Board Member of Scientific Reports. His present research efforts are in the areas of nanophotonics, optoelectronics, optomechanics, and nanoelectromechanics.

Hon Ki Tsang (M'91-SM'04-F'19) received the Bachelor of Arts (Hons.) degree in Engineering and the Ph.D. degree from the University of Cambridge. In 1990 and 1993, he was a Research Visitor at Bellcore (Redbank, NJ, USA). He was also a Postdoctoral Fellow at the School of Physics, University of Bath during 1990-1993. He joined The Chinese University of Hong Kong in 1993 as a Lecturer. He served as Chairman of the Department of Electronic Engineering from 2010 to 2016. He was Editor-in-Chief of the IEEE Photonics Society Newsletter 2012-2014. He is currently the Editor-in-Chief of IEEE Journal of Quantum Electronics. He has published more than 160 papers in journals and over 200 papers in conferences. He is an OSA Fellow and an IEEE Fellow. His research interests include photonic integrated circuits, silicon photonics, nonlinear waveguides, hybrid integration of two-dimensional materials, optical communications, and integrated quantum photonics. 